\title{
Numerical Approaches to Spacetime Singularities
}

\author{
Beverly K. Berger \\ Physics Department, Oakland University, Rochester, MI 48309 USA \\ http://www.oakland.edu/ ${ }^{\sim}$ berger \\ and \\ Physics Division, National Science Foundation \\ 4201 Wilson Blvd., Arlington, VA 22230 USA \\ bberger@nsf.gov \\ Published on 14 January 2002 \\ www.livingreviews.org/Articles/Volume5/2002-1berger \\ Living Reviews in Relativity \\ Published by the Max Planck Institute for Gravitational Physics \\ Albert Einstein Institute, Germany
}

\begin{abstract}
This Living Review updates a previous version [2.5] which is itself an update of a review article [3]]. Numerical exploration of the properties of singularities could, in principle, yield detailed understanding of their nature in physically realistic cases. Examples of numerical investigations into the formation of naked singularities, critical behavior in collapse, passage through the Cauchy horizon, chaos of the Mixmaster singularity, and singularities in spatially inhomogeneous cosmologies are discussed.
\end{abstract}

(c)2002 Max-Planck-Gesellschaft and the authors. Further information on copyright is given at http://www.livingreviews.org/Info/Copyright/. For permission to reproduce the article please contact livrev@aei-potsdam.mpg.de. 


\section{Article Amendments}

On author request a Living Reviews article can be amended to include errata and small additions to ensure that the most accurate and up-to-date information possible is provided. For detailed documentation of amendments, please go to the article's online version at

http://www. livingreviews.org/Articles/Volume5/2002-1berger/.

Owing to the fact that a Living Reviews article can evolve over time, we recommend to cite the article as follows:

Berger, B.K,

"Numerical Approaches to Spacetime Singularities",

Living Rev. Relativity, 5, (2002), 1. [Online Article]: cited on <date>, http://www.livingreviews.org/Articles/Volume5/2002-1berger/.

The date in 'cited on <date>' then uniquely identifies the version of the article you are referring to. 


\section{Contents}

IIntroduction 4

$\begin{array}{lll}2 & \text { Singularities in AF Spacetimes } & 7\end{array}$

2.1 Naked singularities and the hoop conjecture . . . . . . . . . 7

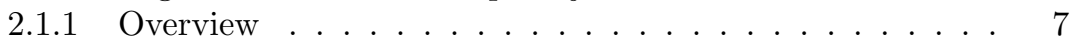

2.1 .2 Naked spindle singularities? . . . . . . . . . . . . . 7

2.1 .3 Recent results . . . . . . . . . . . . . . . . . . . 9 9

2.1 .4 Going further . . . . . . . . . . . . . . . . . . . . . 9 9

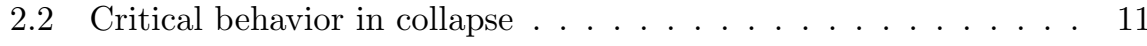

2.2 .1 Gravitational collapse simulations . . . . . . . . . . 11

2.2 .2 Critical solutions as an eigenvalue problem . . . . . . . 12

2.2 .3 Recent results . . . . . . . . . . . . . . . . . . . . . 13

2.2 .4 Going further . . . . . . . . . . . . . . . . . . . . . . 14

2.3 Nature of the singularity in charged or rotating black holes . . . 15

2.3$.$] Overview . . . . . . . . . . . . . . . 15$

2.3 .2 Numerical studies $\ldots \ldots \ldots \ldots \ldots$

2.3 .3 Going further $\ldots \ldots \ldots \ldots \ldots \ldots$

3 Singularities in Cosmological Models $\quad 19$

3.1 Singularities in spatially homogeneous cosmologies . . . . . . . 19

B.2 Numerical methods . . . . . . . . . . . . . . . . . . . . . . . 19

3.2.1 Symplectic methods . . . . . . . . . . . . . . . . . . . . 19

3.2.2 Other methods . . . . . . . . . . . . . . . . . . 20

3.3 Mixmaster dynamics $\ldots \ldots \ldots \ldots \ldots \ldots$

3.3.1 Overview . . . . . . . . . . . . . . . . . . 21

3.3 .2 Recent developments . . . . . . . . . . . . . . . . . 23

3.3 .3 Going further $\ldots \ldots \ldots \ldots \ldots$

3.4 Inhomogeneous cosmologies . . . . . . . . . . . . . . . 24

3.4. Overview . . . . . . . . . . . . . . . . . 24

3.4 .2 Gowdy cosmologies and their generalizations . . . . . . 25

$3.4 .3 \quad U(1)$ symmetric cosmologies . . . . . . . . . . . . . . . 27

3.4 .4 Going further $\ldots \ldots \ldots \ldots$. . . . . . . . . . . . 27

$\begin{array}{ll}4 \text { Discussion } & 30\end{array}$

5 Acknowledgements 31

References 32 


\section{Introduction}

The singularity theorems [245, [139, 140, 114] state that Einstein's equations will not evolve generic, regular initial data arbitrarily far into the future or the past. An obstruction such as infinite curvature or the termination of geodesics will always arise to stop the evolution somewhere. The simplest, physically relevant solutions representing for example a homogeneous, isotropic universe (Friedmann-Robertson-Walker (FRW)) or a spherically symmetric black hole (Schwarzschild) contain space-like infinite curvature singularities. Although, in principle, the presence of a singularity could lead to unpredictable measurements for a physically realistic observer, this does not happen for these two solutions. The surface of last scattering of the cosmic microwave background in the cosmological case and the event horizon in the black hole (BH) case effectively hide the singularity from present day, external observers. The extent to which this "hidden" singularity is generic and the types of singularities that appear in generic spacetimes remain major open questions in general relativity. The questions arise quickly since other exact solutions to Einstein's equations have singularities which are quite different from those described above. For example, the charged BH (Reissner-Nordström solution) has a time-like singularity. It also contains a Cauchy horizon $(\mathrm{CH})$ marking the boundary of predictability of space-like initial data supplied outside the BH. A test observer can pass through the $\mathrm{CH}$ to another region of the extended spacetime. More general cosmologies can exhibit singularity behavior different from that in FRW. The Big Bang in FRW is classified as an asymptotically velocity term dominated (AVTD) singularity [94, [65] since any spatial curvature term in the Hamiltonian constraint becomes negligible compared to the square of the expansion rate as the singularity is approached. However, some anisotropic, homogeneous models exhibit Mixmaster dynamics (MD) [22, 187] and are not AVTD - the influence of the spatial scalar curvature can never be neglected. For more rigorous discussions of the classification and properties of the types of singularities see [97, 240]].

Once the simplest, exactly solvable models are left behind, understanding of the singularity becomes more difficult. There has been significant analytic progress [244, 1191, 21.9, 3]. However, until recently such methods have yielded either detailed knowledge of unrealistic, simplified (usually by symmetries) spacetimes or powerful, general results that do not contain details. To overcome these limitations, one might consider numerical methods to evolve realistic spacetimes to the point where the properties of the singularity may be identified. Of course, most of the effort in numerical relativity applied to $\mathrm{BH}$ collisions has addressed the avoidance of singularities [100]. One wishes to keep the computational grid in the observable region outside the horizon. Much less computational effort has focused on the nature of the singularity itself. Numerical calculations, even more than analytic ones, require finite values for all quantities. Ideally then, one must describe the singularity by the asymptotic non-singular approach to it. A numerical method which can follow the evolution into this asymptotic regime will then yield information about the singularity. Since the numerical study must begin with a particular set of initial data, the results can never have

Living Reviews in Relativity (2002-1)

http://www.livingreviews.org 
the force of mathematical proof. One may hope, however, that such studies will provide an understanding of the "phenomenology" of singularities that will eventually guide and motivate rigorous results. Some examples of the interplay between analytic and numerical results and methods will be given here.

In the following, we shall consider examples of numerical study of singularities both for asymptotically flat (AF) spacetimes and for cosmological models. These examples have been chosen to illustrate primarily numerical studies whose focus is the nature of the singularity itself. In the AF context, we shall consider two questions:

The first is whether or not naked singularities exist for realistic matter sources. One approach has been to explore highly non-spherical collapse looking for spindle or pancake singularities. If the formation of an event horizon requires a limit on the aspect ratio of the matter [2.3.9], such configurations may yield a naked singularity. Analytic results suggest that one must go beyond the failure to observe an apparent horizon to conclude that a naked singularity has formed [244]. Another approach is to probe the limits between initial configurations which lead to black holes and those which yield no singularity at all (i.e. flat spacetime plus radiation) to explore the singularity as the $\mathrm{BH}$ mass goes to zero. This quest led naturally to the discovery of critical behavior in the collapse of a scalar field [78]. In the initial study, the critical (Choptuik) solution is a zero mass naked singularity (visible from null infinity). It is a counterexample to the cosmic censorship conjecture [1.35]. However, it is a non-generic one since fine-tuning of the initial data is required to produce this critical solution. In a possibly related study, Christodoulou has shown [81] that for the spherically symmetric Einstein-scalar field equations, there always exists a perturbation that will convert a solution with a naked singularity (but of a different class from Choptuik's) to one with a black hole. Reviews of critical phenomena in gravitational collapse can be found in [46, 125, ॠ30, 126].

The second question which is now beginning to yield to numerical attack involves the stability of the Cauchy horizon in charged or rotating black holes. It has been conjectured [245, [7.3] that a real observer, as opposed to a test mass, cannot pass through the $\mathrm{CH}$ since realistic perturbed spacetimes will convert the $\mathrm{CH}$ to a strong spacelike singularity [240]. Numerical studies [56, 92, 63] show that a weak, null singularity forms first as had been predicted [212, 2012].

In cosmology, we shall consider both the behavior of the Mixmaster model and the issue of whether or not its properties are applicable to generic cosmological singularities. Although numerical evolution of the Mixmaster equations has a long history, developments in the past decade were motivated by inconsistencies between the known sensitivity to initial conditions and standard measures of the chaos usually associated with such behavior [193, 22.3, 22.5, 28, 102, 62, 1447, 216]. A coordinate invariant characterization of Mixmaster chaos has been formulated [86] which, while criticized in its details [194], has essentially resolved the question. In addition, a new extremely fast and accurate algorithm for Mixmaster simulations has been developed [38].

Belinskii, Khalatnikov, and Lifshitz (BKL) long ago claimed [17, 18, 1.9, 222, [2] that it is possible to formulate the generic cosmological solution to Einstein's

Living Reviews in Relativity (2002-1)

http://www.livingreviews.org 
equations near the singularity as a Mixmaster universe at every spatial point. While others have questioned the validity of this claim [I3], numerical evidence has been obtained for oscillatory behavior in the approach to the singularity of spatially inhomogeneous cosmologies [250, 43, 36, 41]. We shall discuss results from a numerical program to address this issue [42, 36, 32]. The key claim by BKL is that sufficiently close to the singularity, each spatial point evolves as a separate universe - most generally of the Mixmaster type. For this to be correct, the dynamical influence of spatial derivatives (embodying communication between spatial points) must be overwhelmed by the time dependence of the local dynamics. In the past few years, numerical simulations of collapsing, spatially inhomogeneous cosmological spacetimes have provided strong support for the BKL picture [42, 35, 44, [250, 43, 36, 41]]. In addition, the Method of Consistent Potentials (MCP) [12.3, 36] has been developed to explain how the BKL asymptotic state arises during collapse. New asymptotic methods have been used to prove that open sets exist with BKL's local behavior (although these are AVTD rather than of the Mixmaster type) [163, 1773, 3]. Recently, van Elst, Uggla, and Wainwright developed a dynamical systems approach to $G_{2}$ cosmologies (i.e. those with 2 spatial symmetries) [242].

Living Reviews in Relativity (2002-1)

http://www.livingreviews.org 


\section{Singularities in AF Spacetimes}

While I have divided this topic into three subsections, there is considerable overlap. The primary questions can be formulated as the cosmic censorship conjecture. The weak cosmic censorship conjecture [20.9] requires a singularity formed from regular, asymptotically flat initial data to be hidden from an external observer by an event horizon. An excellent review of the meaning and status of weak cosmic censorship has been given by Wald [244]. Counter examples have been known for a long time but tend to be dismissed as unrealistic in some way. The strong form of the cosmic censorship conjecture [210] forbids timelike singularities, even within black holes.

\subsection{Naked singularities and the hoop conjecture}

\subsubsection{Overview}

Perhaps, the first numerical approach to study the cosmic censorship conjecture consisted of attempts to create naked singularities. Many of these studies were motivated by Thorne's "hoop conjecture" [2:3.] that collapse will yield a black hole only if a mass $M$ is compressed to a region with circumference $C \leq 4 \pi M$ in all directions. (As is discussed by Wald [244], one runs into difficulties in any attempt to formulate the conjecture precisely. For example, how does one define $C$ and $M$, especially if the initial data are not at least axially symmetric? Schoen and Yau defined the size of an arbitrarily shaped mass distribution in [[2Z8]. A non-rigorous prescription was used in a numerical study by Chiba [75].) If the hoop conjecture is true, naked singularities may form if collapse can yield $C \geq 4 \pi M$ in some direction. The existence of a naked singularity is inferred from the absence of an apparent horizon (AH) which can be identified locally. Although a definitive identification of a naked singularity requires the event horizon (EH) to be proven to be absent, to identify an $\mathrm{EH}$ requires knowledge of the entire spacetime. While one finds an $\mathrm{AH}$ within an $\mathrm{EH}$ [166, [67], it is possible to construct a spacetime slicing which has no $\mathrm{AH}$ even though an EH is present [246]. Methods to find an EH in a numerically determined spacetime have only recently become available and have not been applied to this issue [179, 184]. A local prescription, applicable numerically, to identify an "isolated horizon" is under development by Ashtekar et al. (see for example [ [ $]$ ]).

\subsubsection{Naked spindle singularities?}

In the best known attempt to produce naked singularities, Shapiro and Teukolsky (ST) [2:30] considered collapse of prolate spheroids of collisionless gas. (Nakamura and Sato [1.95] had previously studied the collapse of non-rotating deformed stars with an initial large reduction of internal energy and apparently found spindle or pancake singularities in extreme cases.) ST solved the general relativistic Vlasov equation for the particles along with Einstein's equations for

Living Reviews in Relativity (2002-1)

http://www.livingreviews.org 


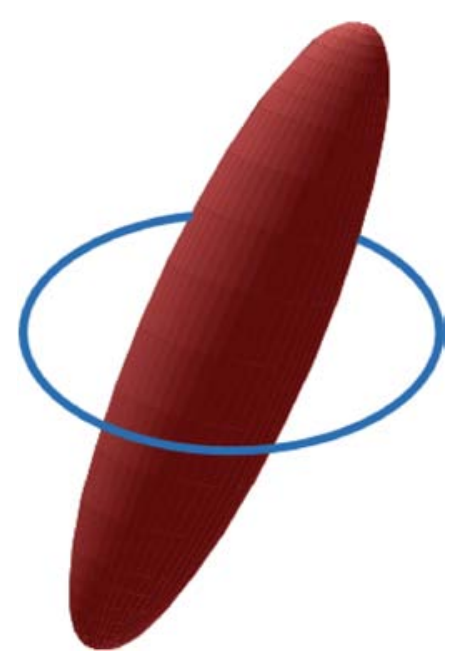

Figure 1: Heuristic illustration of the hoop conjecture.

the gravitational field. They then searched each spatial slice for trapped surfaces. If no trapped surfaces were found, they concluded that there was no AH in that slice. The curvature invariant $I=R_{\mu \nu \rho \sigma} R^{\mu \nu \rho \sigma}$ was also computed. They found that an $\mathrm{AH}$ (and presumably a $\mathrm{BH}$ ) formed if $C \leq 4 \pi M<1$ everywhere, but no $\mathrm{AH}$ (and presumably a naked singularity) in the opposite case. In the latter case, the evolution (not surprisingly) could not proceed past the moment of formation of the singularity. In a subsequent study, ST [231] also showed that a small amount of rotation (counter rotating particles with no net angular momentum) does not prevent the formation of a naked spindle singularity. However, Wald and Iyer [246] have shown that the Schwarzschild solution has a time slicing whose evolution approaches arbitrarily close to the singularity with no $\mathrm{AH}$ in any slice (but, of course, with an $\mathrm{EH}$ in the spacetime). This may mean that there is a chance that the increasing prolateness found by ST in effect changes the slicing to one with no apparent horizon just at the point required by the hoop conjecture. While, on the face of it, this seems unlikely, Tod gives an example where a trapped surface does not form on a chosen constant time slice - but rather different portions form at different times. He argues that a numerical simulation might be forced by the singularity to end before the formation of the trapped surface is complete. Such a trapped surface would not be found by the simulations [241]. In response to such a possibility, Shapiro and Teukolsky considered equilibrium sequences of prolate relativistic star clusters [232]. The idea is to counter the possibility that an EH might form after the time when the simulation must stop. If an equilibrium configuration is non-singular, it cannot contain an EH since singularity theorems say that an EH implies a singularity. However, a sequence of non-singular equilibria with rising $I$ ever closer to the spindle singularity would lend support to the existence of a naked spindle singularity since one can approach the singular state without formation of an

Living Reviews in Relativity (2002-1)

http://www.livingreviews.org 
EH. They constructed this sequence and found that the singular end points were very similar to their dynamical spindle singularity. Wald believes, however, that it is likely that the ST slicing is such that their singularities are not naked - a trapped surface is present but has not yet appeared in their time slices [244].

Another numerical study of the hoop conjecture was made by Chiba et al. [66]. Rather than a dynamical collapse model, they searched for AH's in analytic initial data for discs, annuli, and rings. Previous studies of this type were done by Nakamura et al. [196] with oblate and prolate spheroids and by Wojtkiewicz [25I] with axisymmetric singular lines and rings. The summary of their results is that an $\mathrm{AH}$ forms if $C \leq 4 \pi M \leq 1.26$. (Analytic results due to Barrabès et al. [10, 9$]$ and Tod [24I] give similar quantitative results with different initial data classes and (possibly) an alternative definition of $C$.)

There is strong analytical evidence against the development of spindle singularities. It has been shown by Chruściel and Moncrief that strong cosmic censorship holds in AF electrovac solutions which admit a $G_{2}$ symmetric Cauchy surface [34]. The evolutions of these highly nonlinear equations are in fact nonsingular.

\subsubsection{Recent results}

Garfinkle and Duncan [114] report preliminary results on the collapse of prolate configurations of Brill waves [60]. They use their axisymmetric code to explore the conjecture of Abrahams et al [8] that prolate configurations with no $\mathrm{AH}$ but large $I$ in the initial slice will evolve to form naked singularities. Garfinkle and Duncan find that the configurations become less prolate as they evolve suggesting that black holes (rather than naked singularities) will form eventually from this type of initial data. Similar results have also been found by Hobill and Webster [149].

Pelath et al. [208] set out to generalize previous results [246, [241] that formation of a singularity in a slice with no AH did not indicate the absence of an EH. They looked specifically at trapped surfaces in two models of collapsing null dust, including the model considered by Barrabès et al. [10]. They indeed find a natural spacetime slicing in which the singularity forms at the poles before the outermost marginally trapped surface (OMTS) (which defines the AH) forms at the equator. Nonetheless, they also find that whether or not an OMTS forms in a slice closely (or at least more closely than one would expect if there were no relevance to the hoop conjecture) follows the predictions of the hoop conjecture.

\subsubsection{Going further}

Motivated by ST's results [2:30], Echeverria [96] numerically studied the properties of the naked singularity that is known to form in the collapse of an infinite, cylindrical dust shell [239]. While the asymptotic state can be found analytically, the approach to it must be followed numerically. The analytic asymptotic solution can be matched to the numerical one (which cannot be followed all the way to the collapse) to show that the singularity is strong (an observer

Living Reviews in Relativity (2002-1)

http://www.livingreviews.org 


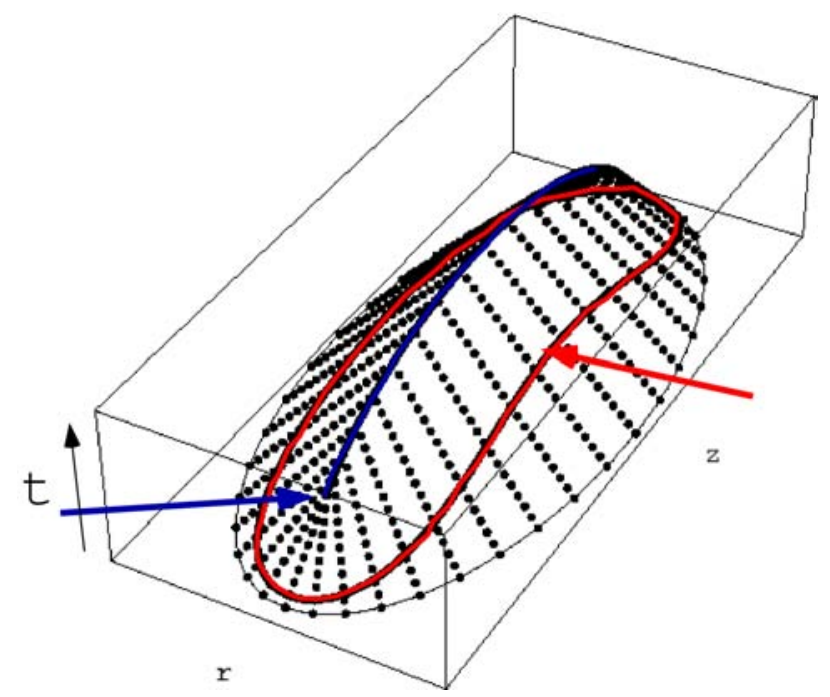

Figure 2: This figure is based on Figure 1 of [208]. The vertical axis is time. The blue curve shows the singularity and the red curve the outermost marginally trapped surface. Note that the singularity forms at the poles (indicated by the blue arrow) before the outermost marginally trapped surface forms at the equator (indicated by the red arrow).

experiences infinite stretching parallel to the symmetry axis and squeezing perpendicular to the symmetry axis). A burst of gravitational radiation emitted just prior to the formation of the singularity stretches and squeezes in opposite directions to the singularity. This result for dust conflicts with rigorously nonsingular solutions for the electrovac case [34]. One wonders then if dust collapse gives any information about singularities of the gravitational field.

One useful result from dust collapse has been the study of gravitational waves which might be associated with the formation of a naked singularity. Such a program has been carried out by Harada, Iguchi, and Nakao [160, 16], 1.98, 1137, [138, [15.9].

Nakamura et al. (NSN) [1.97] conjectured that even if naked spindle singularities could exist, they would either disappear or become black holes. This demise of the naked singularity would be caused by the back reaction of the gravitational waves emitted by it. While NSN proposed a numerical test of their conjecture, they believed it to be beyond the current generation of computer technology.

Chiba [75] extended previous results [76] to search for AH's in spacetimes without axisymmetry but with a discrete symmetry. The discrete symmetry is used to identify an analog of a symmetry axis to allow a prescription for an analog of the circumference. Given this construction, it is possible to formulate the hoop conjecture in this case and to explore its validity in numerically

Living Reviews in Relativity (2002-1)

http://www.livingreviews.org 
constructed momentarily static spacetimes. Explicit application was made to multiple black holes distributed along a ring. It was found that, if the quantity $\mathcal{C}$ defined as the circumference is less than approximately 1.168 , a common apparent horizon surrounds the multi-black-hole configuration.

The results of all these searches for naked spindle singularities are controversial but could be resolved if the presence or absence of the $\mathrm{EH}$ could be determined. One could demonstrate numerically whether or not Wald's view of ST's results is correct by using existing EH finders [17.9, 184] in a relevant simulation. Of course, this could only be effective if the simulation covered enough of the spacetime to include (part of) the EH.

\subsection{Critical behavior in collapse}

\subsubsection{Gravitational collapse simulations}

For a more detailed discussion of critical behavior see [126]. Since Gundlach's Living Review article has appeared, the updates in this section will be restricted to results I find especially interesting.

Critical behavior was originally found by Choptuik [78] in a numerical study of the collapse of a spherically symmetric massless scalar field. For recent reviews see [12.5, [130]. We note that this is the first completely new phenomenon in general relativity to be discovered by numerical simulation. In collapse of a scalar field, essentially two things can happen: Either a black hole (BH) forms or the scalar waves pass through each other and disperse. Choptuik discovered that for any 1-parameter set of initial data labeled by $p$, there is a critical value $p^{*}$ such that $p>p^{*}$ yields a $\mathrm{BH}$. He found

$$
M_{\mathrm{BH}} \approx C_{\mathrm{F}}\left(p-p^{*}\right)^{\gamma},
$$

where $M_{\mathrm{BH}}$ is the mass of the eventual $\mathrm{BH}$. The constant $C_{\mathrm{F}}$ depends on the parameter of the initial data that is selected but $\gamma \approx 0.37$ is the same for all choices. Furthermore, in terms of logarithmic variables $\rho=\ln r+\kappa, \tau=$ $\ln \left(T_{0}^{*}-T_{0}\right)+\kappa\left(T_{0}\right.$ is the proper time of an observer at $r=0$, where $r$ is the radial coordinate, with $T_{0}^{*}$ the finite proper time at which the critical evolution concludes, and $\kappa$ is a constant which scales $r$ ), the waveform $X$ repeats (echoes) at intervals of $\Delta$ in $\tau$ if $\rho$ is rescaled to $\rho-\Delta$, i.e. $X(\rho-\Delta, \tau-\Delta) \approx X(\rho, \tau)$. The scaling behavior (11) demonstrates that the minimum BH mass (for bosons) is zero. The critical solution itself is a counter-example to cosmic censorship (since the formation of the zero mass $\mathrm{BH}$ causes high curvature regions to become visible at $r=\infty$ ). (See, e.g., the discussion in Hirschmann and Eardley [145].) The numerical demonstration of this feature of the critical solution was provided by Hamadé and Stewart [135]. This result caused Hawking to pay off a bet to Preskill and Thorne [6], ए70].

Soon after this discovery, scaling and critical phenomena were found in a variety of contexts. Abrahams and Evans [I] discovered the same phenomenon in axisymmetric gravitational wave collapse with a different value of $\Delta$ and, to within numerical error, the same value of $\gamma$. (Note that the rescaling of

Living Reviews in Relativity (2002-1)

http://www.livingreviews.org 


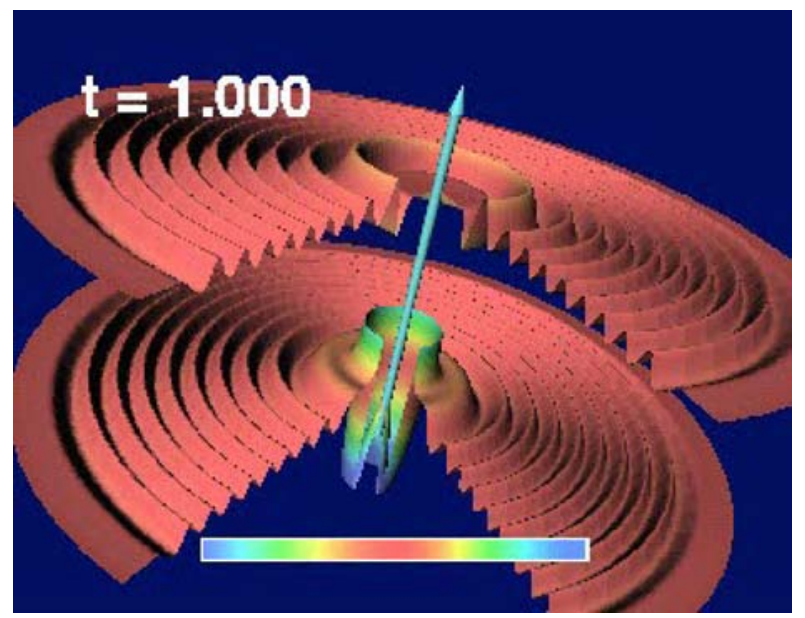

Figure 3: This figure is the final frame of an animation of Type II critical behavior in Einstein-Yang-Mills collapse. Note the echoing in the near-critical solution. For the entire movie and related references see [77].

$r$ with $e^{\Delta} \approx 30$ required Choptuik to use adaptive mesh refinement (AMR) to distinguish subsequent echoes. Abrahams and Evans' smaller $\Delta\left(e^{\Delta} \approx 1.8\right)$ allowed them to see echoing with their $2+1$ code without AMR.) Garfinkle [110] confirmed Choptuik's results with a completely different algorithm that does not require AMR. He used Goldwirth and Piran's [ए19] method of simulating Christodoulou's [ $[80]$ formulation of the spherically symmetric scalar field in null coordinates. This formulation allowed the grid to be automatically rescaled by choosing the edge of the grid to be the null ray that just hits the central observer at the end of the critical evolution. (Missing points of null rays that cross the central observer's world line are replaced by interpolation between those that remain.) Hamadé and Stewart [135] have also repeated Choptuik's calculation using null coordinates and AMR. They are able to achieve greater accuracy and find $\gamma \approx 0.374$.

\subsubsection{Critical solutions as an eigenvalue problem}

Evans and Coleman [98] realized that self-similar rather than self-periodic collapse might be more tractable both numerically (since ODE's rather than PDE's are involved) and analytically. They discovered that a collapsing radiation fluid had that desirable property. (Note that self-similarity (homothetic motion) is incompatible with AF [93, 108]. However, most of the action occurs in the center so that a match of the self-similar inner region to an outer AF one should always be possible.) In a series of papers, Hirschmann and Eardley [144, 1145] developed a (numerical) self-similar solution to the spherically symmetric complex scalar field equations. These are ODE's with too many boundary conditions causing a solution to exist only for certain fixed values of $\Delta$. Numerical solution of this

Living Reviews in Relativity (2002-1)

http://www.livingreviews.org 
eigenvalue problem allows very accurate determination of $\Delta$. The self-similarity also allows accurate calculation of $\gamma$ as follows: The critical $p=p^{*}$ solution is unstable to a small change in $p$. At any time $t$ (where $t<0$ is increasing toward zero), the amplitude $a$ of the perturbation exhibits power law growth:

$$
a \propto\left(p-p^{*}\right)(-t)^{-\kappa}
$$

where $\kappa>0$. At any fixed $t$, larger $a$ implies larger $M_{\mathrm{BH}}$. Equivalently, any fixed amplitude $a=\delta$ will be reached faster for larger eventual $M_{\mathrm{BH}}$. Scaling arguments give the dependence of $M_{\mathrm{BH}}$ on the time at which any fixed amplitude is reached:

$$
M_{\mathrm{BH}} \propto\left(-t_{1}\right)
$$

where

$$
\left(p-p^{*}\right)\left(-t_{1}\right)^{\kappa} \propto \delta
$$

Thus

$$
M_{\mathrm{BH}} \propto\left(p-p^{*}\right)^{1 / \kappa}
$$

Therefore, one need only identify the growth rate of the unstable mode to obtain an accurate value of $\gamma=1 / \kappa$. It is not necessary to undertake the entire dynamical evolution or probe the space of initial data. (While this procedure allowed Hirschmann and Eardley to obtain $\gamma=0.387106$ for the complex scalar field solution, they later found [146] that, in this regime, the complex scalar field has 3 unstable modes. This means [129, ए26] that the Eardley-Hirschmann solution is not a critical solution. A perturbation analysis indicates that the critical solution for the complex scalar field is the discretely self-similar one found for the real scalar field [[29].) Koike et al. [ए76] obtain $\gamma=0.35580192$ for the EvansColeman solution. Although the similarities among the critical exponents $\gamma$ in the collapse computations suggested a universal value, Maison [183] used these same scaling-perturbation methods to show that $\gamma$ depends on the equation of state $p=k \rho$ of the fluid in the Evans-Coleman solution. Gundlach [127] used a similar approach to locate Choptuik's critical solution accurately. This is much harder due to its discrete self-similarity. He reformulates the model as nonlinear hyperbolic boundary value problem with eigenvalue $\Delta$ and finds $\Delta=3.4439$. As with the self-similar solutions described above, the critical solution is found directly without the need to perform a dynamical evolution or explore the space of initial data. Hara et al. extended the renormalization group approach of [ए76] and applied it to the continuously-self-similar case [1.36]. (For an application of renormalization group methods to cosmology see [162].)

\subsubsection{Recent results}

Gundlach [I2.9] completed his eigenvalue analysis of the Choptuik solution to find the growth rate of the unstable mode to be $\gamma=0.374 \pm 0.001$. He also predicted a periodic "wiggle" in the Choptuik mass scaling relation. This was later observed numerically by Hod and Piran [152]. Self-similar critical behavior has

Living Reviews in Relativity (2002-1)

http://www.livingreviews.org 
been seen in string theory related axion-dilaton models [95, 1334] and in the nonlinear $\sigma$-model [146]. Garfinkle and Duncan have shown that subcritical collapse of a spherically symmetric scalar field yields a scaling relation for the maximum curvature observed by the central observer with critical parameters that would be expected on the basis of those found for supercritical collapse [113].

Choptuik et al. [7.9] have generalized the original Einstein-scalar field calculations to the Einstein-Yang-Mills (EYM) (for $S U(2)$ ) case. Here something new was found. Two types of behavior appeared depending on the initial data. In Type I, BH formation had a non-zero mass threshold. The critical solution is a regular, unstable solution to the EYM equations found previously by Bartnik and McKinnon [14]. In Type II collapse, the minimum BH mass is zero with the critical solution similar to that of Choptuik (with a different $\gamma \approx 0.20$, $\Delta \approx 0.74$ ). Gundlach has also looked at this case with the same results [ए28]. The Type I behavior arises when the collapsing system has a metastable static solution in addition to the Choptuik critical one [1.32].

Brady, Chambers and Gonçalves [ [71, 52] conjectured that addition of a mass to the scalar field of the original model would break scale invariance and might yield a distinct critical behavior. They found numerically the same Type I and II "phases" seen in the EYM case. The Type II solution can be understood as perturbations of Choptuik's original model with a small scalar field mass $\mu$. Here small means that $\lambda \mu<<1$ where $\lambda$ is the spatial extent of the original nonzero field region. (The scalar field is well within the Compton wavelength corresponding to $\mu$.) On the other hand, $\lambda \mu>>1$ yields Type I behavior. The minimum mass critical solution is an unstable soliton of the type found by Seidel and Suen [229]. The massive scalar field can be treated as collapsing dust to yield a criterion for $\mathrm{BH}$ formation [120]].

The Choptuik solution has also been found to be the critical solution for charged scalar fields [132, 15T]. As $p \rightarrow p^{*}, Q / M \rightarrow 0$ for the black hole. $Q$ obeys a power law scaling. Numerical study of the critical collapse of collisionless matter (Einstein-Vlasov equations) has yielded a non-zero minimum BH mass [217, [201]. Bizoń and Chmaj [47] have considered the critical collapse of skyrmions.

An astrophysical application of BH critical phenomena has been considered by Niemeyer and Jedamzik [19.9] and Yokoyama [2.52]. They consider its implications for primordial BH formation and suggest that it could be important.

\subsubsection{Going further}

The question is then why these critical phenomena should appear in so many collapsing gravitational systems. The discrete self-similarity of Choptuik's solution may be understood as scaling of a limit cycle [136]. (The self-similarity of other systems may be understood as scaling of a limit point.) Garfinkle [ए10] originally conjectured that the scale invariance of Einstein's equations might provide an underlying explanation for the self-similarity and discrete-self-similarity found in collapse and proposed a spacetime slicing which might manifestly show this. In fact, he later showed (with Meyer) [116] that, while the originally proposed

Living Reviews in Relativity (2002-1)

http://www.livingreviews.org 
slicing failed, a foliation based on maximal slicing did make the scaling manifest. These ideas formed the basis of a much more ambitious program by Garfinkle and Gundlach to use underlying actual or approximate symmetries to construct coordinate systems for numerical simulations [15]].

An interesting "toy model" for general relativity in many contexts has been wave maps, also known as nonlinear $\sigma$ models. One of these contexts is critical collapse [146]. Recently and independently, Bizoń et al. [48] and Liebling et al. [180] evolved wave maps from the base space of $3+1$ Minkowski space to the target space $S^{3}$. They found critical behavior separating singular and nonsingular solutions. For some families of initial data, the critical solution is self-similar and is an intermediate attractor. For others, a static solution separates the singular and nonsingular solutions. However, the static solution has several unstable modes and is therefore not a critical solution in the usual sense. Bizoń and Tabor [49] have studied Yang-Mills fields in $D+1$ dimensions and found that generic solutions with sufficiently large initial data blow up in a finite time and that the mechanism for blowup depends on $D$. Husa et al. [158] then considered the collapse of $S U(2)$ nonlinear sigma models coupled to gravity and found a discretely self-similar critical solution for sufficiently large dimensionless coupling constant. They also observe that for sufficiently small coupling constant values, there is a continuously self-similar solution. Interestingly, there is an intermediate range of coupling constant where the discrete self-similarity is intermittent [2.38].

Until recently, only Abrahams and Evans [T] had ventured beyond spherical symmetry. The first additional departure has been made by Gundlach [131]. He considered spherical and non-spherical perturbations of $P=\frac{1}{3} \rho$ perfect fluid collapse. Only the original (spherical) growing mode survived.

Recently, critical phenomena have been explored in $2+1$ gravity. Pretorius and Choptuik [2.5] numerically evolved circularly symmetric scalar field collapse in $2+1$ anti-de Sitter space. They found a continuously self-similar critical solution at the threshold for black hole formation. The BH's which form have BTZ [8] exteriors with strong curvature, spacelike singularities in the interior. Remarkably, Garfinkle obtained an analytic critical solution by assuming continuous self-similarity which agrees quite well with the one obtained numerically [एँ2].

\subsection{Nature of the singularity in charged or rotating black holes}

\subsubsection{Overview}

Unlike the simple singularity structure of the Schwarzschild solution, where the event horizon encloses a spacelike singularity at $r=0$, charged and/or rotating BH's have a much richer singularity structure. The extended spacetimes have an inner Cauchy horizon $(\mathrm{CH})$ which is the boundary of predictability. To the future of the $\mathrm{CH}$ lies a timelike (ring) singularity [245]. Poisson and Israel [212, [213] began an analytic study of the effect of perturbations on the $\mathrm{CH}$. Their goal

Living Reviews in Relativity (2002-1)

http://www.livingreviews.org 
was to check conjectures that the blue-shifted infalling radiation during collapse would convert the $\mathrm{CH}$ into a true singularity and thus prevent an observer's passage into the rest of the extended regions. By including both ingoing and back-scattered outgoing radiation, they find for the Reissner-Nordström (RN) solution that the mass function (qualitatively $R_{\alpha \beta \gamma \delta} \propto M / r^{3}$ ) diverges at the $\mathrm{CH}$ (mass inflation). However, Ori showed both for RN and Kerr [2022, 2013] that the metric perturbations are finite (even though $R_{\mu \nu \rho \sigma} R^{\mu \nu \rho \sigma}$ diverges) so that an observer would not be destroyed by tidal forces (the tidal distortion would be finite) and could survive passage through the $\mathrm{CH}$. A numerical solution of the Einstein-Maxwell-scalar field equations would test these perturbative results.

For an excellent, brief review of the history of this topic see the introduction in [2015].

\subsubsection{Numerical studies}

Gnedin and Gnedin [118] have numerically evolved the spherically symmetric Einstein-Maxwell with massless scalar field equations in a $2+2$ formulation. The initial conditions place a scalar field on part of the RN event horizon (with zero field on the rest). An asymptotically null or spacelike singularity whose shape depends on the strength of the initial perturbation replaces the $\mathrm{CH}$. For a sufficiently strong perturbation, the singularity is Schwarzschild-like. Although they claim to have found that the $\mathrm{CH}$ evolved to become a spacelike singularity, the diagrams in their paper show at least part of the final singularity to be null or asymptotically null in most cases.

Brady and Smith [56] used the Goldwirth-Piran formulation [119] to study the same problem. They assume the spacetime is $\mathrm{RN}$ for $v<v_{0}$. They follow the evolution of the $\mathrm{CH}$ into a null singularity, demonstrate mass inflation, and support (with observed exponential decay of the metric component $g$ ) the validity of previous analytic results [212, 2113, 2012, 20103] including the "weak" nature of the singularity that forms. They find that the observer hits the null $\mathrm{CH}$ singularity before falling into the curvature singularity at $r=0$. Whether or not these results are in conflict with Gnedin and Gnedin [118] is unclear [50]]. However, it has become clear that Brady and Smith's conclusions are correct. The collapse of a scalar field in a charged, spherically symmetric spacetime causes an initial RN CH to become a null singularity except at $r=0$ where it is spacelike. The observer falling into the $\mathrm{BH}$ experiences (and potentially survives) the weak, null singularity [2022, 2013, 51] before the spacelike singularity forms. This has been confirmed by Droz [92] using a plane wave model of the interior and by Burko [6.3] using a collapsing scalar field. See also [6.5, 68]

Numerical studies of the interiors of non-Abelian black holes have been carried out by Breitenlohner et al. [57, 58] and by Gal'tsov et al. [9], 104, 105, 106] (see also [2.54]). Although there appear to be conflicts between the two groups, the differences can be attributed to misunderstandings of each other's notation [5.9]. The main results include an interesting oscillatory behavior of the metric.

The current status of the topic of singularities within BH's includes an appar-

Living Reviews in Relativity (2002-1)

http://www.livingreviews.org 


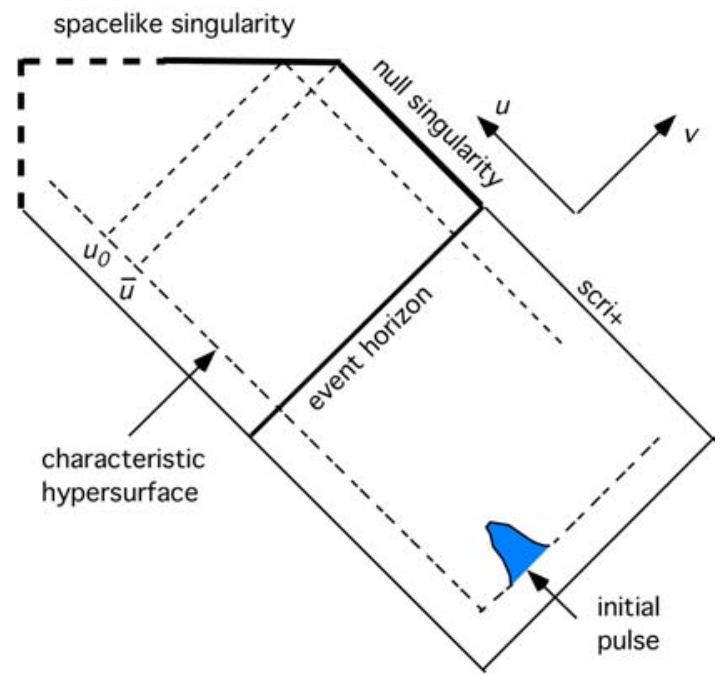

Figure 4: Figure 1 from [[6] is a schematic diagram of the singularity structure within a spherical charged black hole.

ent conflict between the belief [1.9] and numerical evidence [36] that the generic singularity is strong, oscillatory, and spacelike, and analytic evidence that the singularity inside a generic (rotating) $\mathrm{BH}$ is weak, oscillatory (but in a different way), and null [206]. See the discussion at the end of [2016].

Various recent perturbative results reinforce the belief that the singularity within a "realistic" (i.e. one which results from collapse) black hole is of the weak, null type described by Ori [202, 2013]. Brady et al. [54] performed an analysis in the spirit of Belinskii et al. [1.9] to argue that the singularity is of this type. They constructed an asymptotic expansion about the $\mathrm{CH}$ of a black hole formed in gravitational collapse without assuming any symmetry of the perturbed solution. To illustrate their techniques, they also considered a simplified "almost" plane symmetric model. Actual plane symmetric models with weak, null singularities were constructed by Ori [2014].

The best numerical evidence for the nature of the singularity in realistic collapse is Hod and Piran's simulation of the gravitational collapse of a spherically symmetric, charged scalar field [154, 153]. Rather than start with (part of) a RN spacetime which already has a singularity (as in, e.g., [56]), they begin with a regular spacetime and follow its dynamical evolution. They observe mass inflation, the formation of a null singularity, and the eventual formation of a spacelike singularity. Ori argues [2016] that the rotating black hole case is different and that the spacelike singularity will never form. No numerical studies beyond perturbation theory have yet been made for the rotating $\mathrm{BH}$.

Some insight into the conflict between the cosmological results and those from $\mathrm{BH}$ interiors may be found by comparing the approach to the singularity in Gowdy [121] spatially inhomogeneous cosmologies (see Section 3.4.2) with

Living Reviews in Relativity (2002-1) http://www.livingreviews.org 
$T^{3}$ [35] and $S^{2} \times S^{1}$ [प]] spatial topologies. Early in the collapse, the boundary conditions associated with the $S^{2} \times S^{1}$ topology influence the gravitational waveforms. Eventually, however, the local behavior of the two spacetimes becomes qualitatively indistinguishable and characteristic of a (non-oscillatory in this case) spacelike singularity. This may be relevant because the BH environment imposes effective boundary conditions on the metric just as topology does. Unfortunately, no systematic study of the relationship between the cosmological and $\mathrm{BH}$ interior results yet exists.

\subsubsection{Going further}

Replacing the AF boundary conditions with Schwarzchild-de Sitter and RNde Sitter BH's was long believed to yield a counterexample to strong cosmic censorship. (See [185, [186, $2 \pi, \mathbf{1 0}]$ and references therein for background and extended discussions.) The stability of the $\mathrm{CH}$ is related to the decay tails of the radiating scalar field. Numerical studies have determined these to be exponential [ [53, [0, [72] rather than power law as in AF spacetimes [67]. The decay tails of the radiation are necessary initial data for numerical study of $\mathrm{CH}$ stability [56] and are crucial to the development of the null singularity. Analytic studies had indicated that the $\mathrm{CH}$ is stable in RN-de Sitter BH's for a restricted range of parameters near extremality. However, Brady et al. [5.5] have shown (using linear perturbation theory) that, if one includes the backscattering of outgoing modes which originate near the event horizon, the $\mathrm{CH}$ is always unstable for all ranges of parameters. Thus RN-de Sitter BH's appear not to be a counterexample to strong cosmic censorship. Numerical studies are needed to demonstrate the existence of a null singularity at the $\mathrm{CH}$ in nonlinear evolution.

Extension of these studies to AF rotating BH's has yielded the surprising result that the tails are not necessarily power law and differ for different fields. Frame dragging effects appear to be responsible [150]].

As a potentially useful approach to the numerical study of singularities, we consider Hübner's [156, 157, 15.5] numerical scheme to evolve on a conformal compactified grid using Friedrich's formalism [I03]. He considers the spherically symmetric scalar field model in a $2+2$ formulation. So far, this code has been used in this context to locate singularities. It was also used to search for Choptuik's scaling [78] and failed to produce agreement with Choptuik's results [156]. This was probably due to limitations of the code rather than inherent problems with the conformal method.

Living Reviews in Relativity (2002-1)

http://www.livingreviews.org 


\section{Singularities in Cosmological Models}

\subsection{Singularities in spatially homogeneous cosmologies}

The generic singularity in spatially homogeneous cosmologies is reasonably well understood. The approach to it asymptotically falls into two classes. The first, called asymptotically velocity term dominated (AVTD) [94, 165], refers to a cosmology that approaches the Kasner (vacuum, Bianchi I) solution [17]] as $\tau \rightarrow \infty$. (Spatially homogeneous universes can be described as a sequence of homogeneous spaces labeled by $\tau$. Here we shall choose $\tau$ so that $\tau=\infty$ coincides with the singularity.) An example of such a solution is the vacuum Bianchi II model [2:36] which begins with a fixed set of Kasner-like anisotropic expansion rates, and, possibly, makes one change of the rates in a prescribed way (Mixmaster-like bounce) and then continues to $\tau=\infty$ as a fixed Kasner solution. In contrast are the homogeneous cosmologies which display Mixmaster dynamics such as vacuum Bianchi VIII and IX [22, 187, 1:33] and Bianchi $\mathrm{VI}_{0}$ and Bianchi I with a magnetic field [178, 229, 1777]. Jantzen [168] has discussed other examples. Mixmaster dynamics describes an approach to the singularity which is a sequence of Kasner epochs with a prescription, originally due to Belinskii, Khalatnikov, and Lifshitz (BKL) [22], for relating one Kasner epoch to the next. Some of the Mixmaster bounces (era changes) display sensitivity to initial conditions one usually associates with chaos, and in fact Mixmaster dynamics is chaotic [86, 194]. Note that we consider an epoch to be a subunit of an era. In some of the literature, this usage is reversed. The vacuum Bianchi I (Kasner) solution is distinguished from the other Bianchi types in that the spatial scalar curvature ${ }^{3} R$, (proportional to) the minisuperspace (MSS) potential [187, 227], vanishes identically. But ${ }^{3} R$ arises in other Bianchi types due to spatial dependence of the metric in a coordinate basis. Thus an AVTD singularity is also characterized as a regime in which terms containing or arising from spatial derivatives no longer influence the dynamics. This means that the Mixmaster models do not have an AVTD singularity since the influence of the spatial derivatives (through the MSS potential) never disappears - there is no last bounce. A more general review of numerical cosmology has been given by Anninos [4].

\section{$3.2 \quad$ Numerical methods}

\subsubsection{Symplectic methods}

Symplectic numerical methods have proven useful in studies of the approach to the singularity in cosmological models [37]. Symplectic ODE and PDE [III, 18.9] methods comprise a type of operator splitting. An outline of the method (for one degree of freedom) follows. Details of the application to the Gowdy model (PDE's in one space and one time direction) are given elsewhere [42].

For a field $q(t)$ and its conjugate momentum $p(t)$, the Hamiltonian operator splits into kinetic and potential energy sub-Hamiltonians. Thus, for an arbitrary

Living Reviews in Relativity (2002-1)

http://www.livingreviews.org 
potential $V(q)$,

$$
H=\frac{1}{2} p^{2}+V(q)=H_{1}(p)+H_{2}(q) .
$$

If the vector $X=(p, q)$ defines the variables at time $t$, then the time evolution is given by

$$
\frac{d X}{d t}=\{H, X\}_{\mathrm{PB}} \equiv A X,
$$

where \{\}$_{\mathrm{PB}}$ is the Poisson bracket. The usual exponentiation yields an evolution operator

$$
e^{A \Delta t}=e^{A_{1}(\Delta t / 2)} e^{A_{2} \Delta t} e^{A_{1}(\Delta t / 2)}+O\left(\Delta t^{3}\right)
$$

for $A=A_{1}+A_{2}$ being the generator of the time evolution. Higher order accuracy may be obtained by a better approximation to the evolution operator [2:34, 2:35] . This method is useful when exact solutions for the sub-Hamiltonians are known. For the given $H$, variation of $H_{1}$ yields the solution

$$
q=q_{0}+p_{0} \Delta t, \quad p=p_{0},
$$

while that of $H_{2}$ yields

$$
q=q_{0}, \quad p=p_{0}-\left.\frac{d V}{d q}\right|_{q_{0}} \Delta t .
$$

Note that $H_{2}$ is exactly solvable for any potential $V$ no matter how complicated although the required differenced form of the potential gradient may be non-trivial. One evolves from $t$ to $t+\Delta t$ using the exact solutions to the subHamiltonians according to the prescription given by the approximate evolution operator (8). Extension to more degrees of freedom and to fields is straightforward $[42,30]$.

\subsubsection{Other methods}

Symplectic methods can achieve convergence far beyond that of their formal accuracy if the full solution is very close to the exact solution from one of the sub-Hamiltonians. Examples where this is the case are given in [38, 3.5.]. On the other hand, because symplectic algorithms are a type of operator splitting, suboperators can be subject to instabilities that are suppressed by the full operator. An example of this may be found in [41]. Other types of PDE solvers are more effective for such spacetimes. One currently popular method is iterative Crank-Nicholson (see [2:37]) which is, in effect, an implicit method without matrix inversion. It was first applied to numerical cosmology by Garfinkle [II]] and was recently used in this context to evolve $T^{2}$ symmetric cosmologies [41].

As pointed out in [42, 35, 41, 43] , spiky features in collapsing inhomogeneous cosmologies will cause any fixed spatial resolution to become inadequate. Such evolutions are therefore candidates for adaptive mesh refinement such as that implemented by Hern and Stuart [143, 142].

Living Reviews in Relativity (2002-1)

http://www.livingreviews.org 


\subsection{Mixmaster dynamics}

\subsubsection{Overview}

Belinskii, Khalatnikov, and Lifshitz [ $[22]$ (BKL) described the singularity approach of vacuum Bianchi IX cosmologies as an infinite sequence of Kasner [ए7T] epochs whose indices change when the scalar curvature terms in Einstein's equations become important. They were able to describe the dynamics approximately by a map evolving a discrete set of parameters from one Kasner epoch to the next [22, [4]]. For example, the Kasner indices for the power law dependence of the anisotropic scale factors can be parametrized by a single variable $u \geq 1$. BKL determined that

$$
u_{n+1}= \begin{cases}u_{n}-1, & 2 \leq u_{n} \\ \left(u_{n}-1\right)^{-1}, & 1 \leq u_{n} \leq 2 .\end{cases}
$$

The subtraction in the denominator for $1 \leq u_{n} \leq 2$ yields the sensitivity to initial conditions associated with Mixmaster dynamics (MD). Misner [187] described the same behavior in terms of the model's volume and anisotropic shears. A multiple of the scalar curvature acts as an outward moving potential in the anisotropy plane. Kasner epochs become straight line trajectories moving outward along a potential corner while bouncing from one side to the other. A change of corner ends a BKL era when $u \rightarrow(u-1)^{-1}$. Numerical evolution of Einstein's equations was used to explore the accuracy of the BKL map as a descriptor of the dynamics as well as the implications of the map [1093, [223, 225, [28]. Rendall has studied analytically the validity of the BKL map as an approximation to the true trajectories [2]8].

Later, the BKL sensitivity to initial conditions was discussed in the language of chaos [II, [72]. An extended application of Bernoulli shifts and Farey trees was given by Rugh [224] and repeated by Cornish and Levin [85]. However, the chaotic nature of Mixmaster dynamics was questioned when numerical evolution of the Mixmaster equations yielded zero Lyapunov exponents (LE's) [102, 62, [147]. (The LE measures the divergence of initially nearby trajectories. Only an exponential divergence, characteristic of a chaotic system, will yield a positive exponent.) Other numerical studies yielded positive LE's [216]. This issue was resolved when the LE was shown numerically and analytically to depend on the choice of time variable [22.3, [27, 99.]. Although MD itself is well-understood, its characterization as chaotic or not had been quite controversial [148].

LeBlanc et al. [178] have shown (analytically and numerically) that MD can arise in Bianchi $\mathrm{VI}_{0}$ models with magnetic fields (see also [18I]). In essence, the magnetic field provides the wall needed to close the potential in a way that yields the BKL map for $u[2,9]$. A similar study of magnetic Bianchi I has been given by LeBlanc [ए77]. Jantzen has discussed which vacuum and electromagnetic cosmologies could display MD [1168].

Cornish and Levin (CL) [86] identified a coordinate invariant way to characterize MD. Sensitivity to initial conditions can lead to qualitatively distinct outcomes from initially nearby trajectories. While the LE measures the exponential divergence of the trajectories, one can also "color code" the regions of

Living Reviews in Relativity (2002-1)

http://www.livingreviews.org 
initial data space corresponding to particular outcomes. A chaotic system will exhibit a fractal pattern in the colors. CL defined the following set of discrete outcomes: During a numerical evolution, the BKL parameter $u$ is evaluated from the trajectories. The first time $u>7$ appears in an approximately Kasner epoch, the trajectory is examined to see which metric scale factor has the largest time derivative. This defines three outcomes and thus three colors for initial data space. However, one can easily invent prescriptions other than that given by Cornish and Levin [86] which would yield discrete outcomes. The fractal nature of initial data space should be common to all of them. It is not clear how the value of the fractal dimension as measured by Cornish and Levin would be affected. The CL prescription has been criticized because it requires only the early part of a trajectory for implementation [194]. Actually, this is the greatest strength of the prescription for numerical work. It replaces a single representative, infinitely long trajectory by (easier to compute) arbitrarily many trajectory fragments.

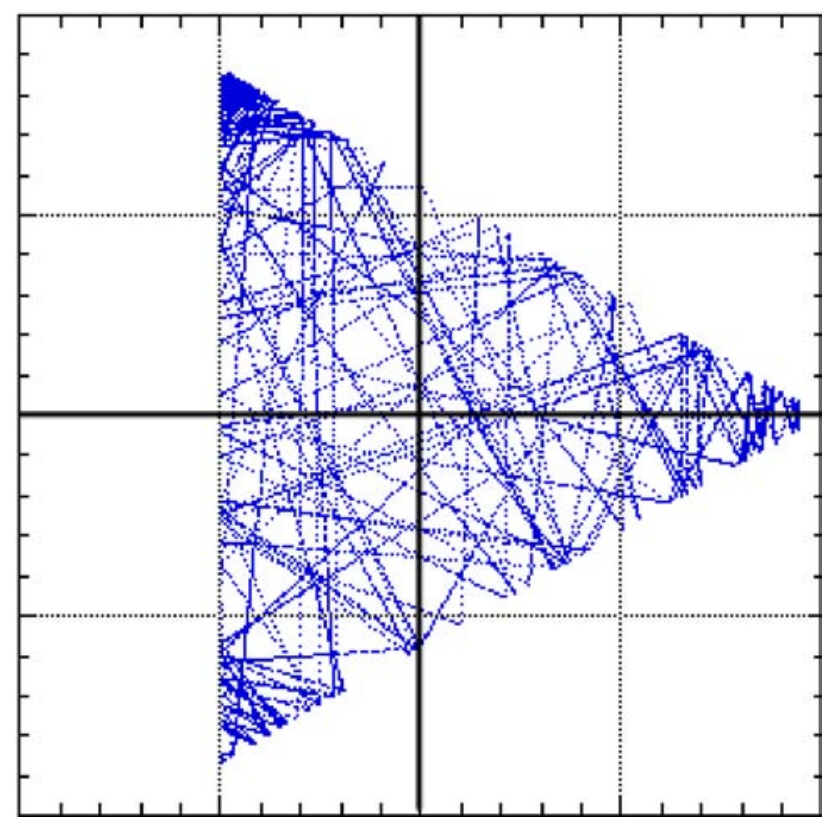

Figure 5: The algorithm of [38] is used to generate a Mixmaster trajectory with more than 250 bounces. The trajectory is shown in the rescaled anisotropy plane with axes $\beta_{ \pm} /|\Omega|$. The rescaling fixes (asymptotically) the location of the bounces.

To study the CL fractal and ergodic properties of Mixmaster evolution [ [ $\underline{6} 6]$, one could take advantage of a new numerical algorithm due to Berger, Garfinkle, and Strasser [38]. Symplectic methods are used to allow the known exact solution for a single Mixmaster bounce [235] to be used in the ODE solver. Standard ODE solvers [214] can take large time steps in the Kasner segments but must

Living Reviews in Relativity (2002-1)

http://www.livingreviews.org 
slow down at the bounce. The new algorithm patches together exactly solved bounces. Tens of orders of magnitude improvement in speed are obtained while the accuracy of machine precision is maintained. In [38], the new algorithm was used to distinguish Bianchi IX and magnetic Bianchi $\mathrm{VI}_{0}$ bounces. This required an improvement of the BKL map (for parameters other than $u$ ) to take into account details of the exponential potential.

So far, most recent effort in MD has focused on diagonal (in the frame of the $S U(2)$ 1-forms) Bianchi IX models. Long ago, Ryan [226] showed that offdiagonal metric components can contribute additional MSS potentials (e.g. a centrifugal wall). This has been further elaborated by Jantzen [169].

\subsubsection{Recent developments}

The most interesting recent developments in spatially homogeneous Mixmaster models have been mathematical. Despite the strong numerical evidence that Bianchi IX, etc. models are well-approximated by the BKL map sufficiently close to the singularity (see, e.g., [38]), there was very little rigorous information on the nature of these solutions. Recently, the existence of a strong singularity (curvature blowup) was proved for Bianchi VIII and IX collapse by Ringström [22I, [222] and for magnetic Bianchi $\mathrm{VI}_{0}$ by Weaver [248]. A remaining open question is how closely an actual Mixmaster evolution is approximated by a single BKL sequence [218, [222]. Since the Berger et al. algorithm [38] achieves machine level accuracy, it can be used to collect numerical evidence on this topic. For example, it has been shown that a given Mixmaster trajectory ceases to track the corresponding sequence of integers obtained from the BKL map (11) at the point where there have been enough era-ending (mixing) bounces to lose all the information encoded in finite precision initial data [38].

\subsubsection{Going further}

There are also numerical studies of Mixmaster dynamics in other theories of gravity. For example, Carretero-Gonzalez et al. [69] find evidence of chaotic behavior in Bianchi IX-Brans-Dicke solutions while Cotsakis et al. [87] have shown that Bianchi IX models in 4th order gravity theories have stable non-chaotic solutions. Barrow and Levin find evidence of chaos in Bianchi IX EinsteinYang-Mills (EYM) cosmologies [एँ]. Their analysis may be applicable to the corresponding EYM black hole interior solutions. Bianchi I models with stringtheory-inspired matter fields have been found by Damour and Henneau [8.9] to have an oscillatory singularity. This is interesting because many other examples exist where matter fields and/or higher dimensions suppress such oscillations (see, e.g., [20] ). Recently, Coley has considered Bianchi IX brane-world models and found them not to be chaotic [84].

Finally, we remark on a successful application of numerical Regge calculus in $3+1$ dimensions. Gentle and Miller have been able to evolve the Kasner solution [117].

Living Reviews in Relativity (2002-1)

http://www.livingreviews.org 


\subsection{Inhomogeneous cosmologies}

\subsubsection{Overview}

BKL have conjectured that one should expect a generic singularity in spatially inhomogeneous cosmologies to be locally of the Mixmaster type (local Mixmaster dynamics (LMD)) [22]. For a review of homogeneous cosmologies, inhomogeneous cosmologies, and the relation between them, see [182]. The main difficulty with the acceptance of this conjecture has been the controversy over whether the required time slicing can be constructed globally [13, [22]. Montani [1.92], Belinskii [16], and Kirillov and Kochnev [ [775, 1774] have pointed out that if the BKL conjecture is correct, the spatial structure of the singularity could become extremely complicated as bounces occur at different locations at different times. BKL seem to imply [22] that LMD should only be expected to occur in completely general spacetimes with no spatial symmetries. However, LMD is actually possible in any spatially inhomogeneous cosmology with a local MSS with a "closed" potential (in the sense of the standard triangular potentials of Bianchi VIII and IX). This closure may be provided by spatial curvature, matter fields, or rotation. A class of cosmological models which appear to have local MD are vacuum universes on $T^{3} \times R$ with a $U(1)$ symmetry [I.90]]. Even simpler plane symmetric Gowdy cosmologies [127, 26] have "open" local MSS potentials. However, these models are interesting in their own right since they have been conjectured to possess an AVTD singularity [12:3]. One way to obtain these Gowdy models is to allow spatial dependence in one direction in Bianchi I homogeneous cosmologies [2][]. It is well-known that addition of matter terms to homogeneous Bianchi I, Bianchi $\mathrm{VI}_{0}$, and other AVTD models can yield Mixmaster behavior [168, ए78, ए77]. Allowing spatial dependence in one direction in such models might then yield a spacetime with LMD. Application of this procedure to magnetic Bianchi $\mathrm{VI}_{0}$ models yields magnetic Gowdy models [250, [247]. Of course, Gowdy cosmologies are not the most general $T^{2}$ symmetric vacuum spacetimes [12], ⒉ 40]]. Restoring the "twists" introduces a centrifugal wall to close the MSS. Magnetic Gowdy and general $T^{2}$ symmetric models appear to admit LMD [247, 248, 41]].

The past few years have seen the development of strong numerical evidence in support of the BKL claims [36]. The Method of Consistent Potentials (MCP) [12:3] has been used to organize the data obtained in simulations of spatially inhomogeneous cosmologies [42, 35, 250, 43, 36, 33, 41]. The main idea is to obtain a Kasner-like velocity term dominated (VTD) solution at every spatial point by solving Einstein's equations truncated by removing all terms containing spatial derivatives. If the spacetime really is AVTD, all the neglected terms will be subdominant (exponentially small in variables where the VTD solution is linear in the time $\tau$ ) when the VTD solution is substituted back into them. For the MCP to successfully predict whether or not the spacetime is AVTD, the dynamics of the full solution must be dominated at (almost) every spatial point by the VTD solution behavior. Surprisingly, MCP predictions have proved valid in numerical simulations of cosmological spacetimes with one [4.3]

Living Reviews in Relativity (2002-1)

http://www.livingreviews.org 
and two [42, 35, 41] spatial symmetries. In the case of $U(1)$ symmetric models, a comparison between the observed behavior [43] and that in a vacuum, diagonal Bianchi IX model written in terms of $U(1)$ variables provides strong support for LMD [45] since the phenomenology of the inhomogeneous cosmologies can be reproduced by this rewriting of the standard Bianchi IX MD.

Polarized plane symmetric cosmologies have been evolved numerically using standard techniques by Anninos, Centrella, and Matzner [5, 6]. The long-term project involving Berger, Garfinkle, and Moncrief and their students to study the generic cosmological singularity numerically has been reviewed in detail elsewhere [37, 36, 32] but will be discussed briefly here.

\subsubsection{Gowdy cosmologies and their generalizations}

The Gowdy model on $T^{3} \times R$ is described by gravitational wave amplitudes $P(\theta, \tau)$ and $Q(\theta, \tau)$ which propagate in a spatially inhomogeneous background universe described by $\lambda(\theta, \tau)$. (We note that the physical behavior of a Gowdy spacetime can be computed from the effect of the metric evolution on a test cylinder [39].) We impose $0 \leq \theta \leq 2 \pi$ and periodic boundary conditions. The time variable $\tau$ measures the area in the symmetry plane with $\tau=\infty$ being a curvature singularity.

Einstein's equations split into two groups. The first is nonlinearly coupled wave equations for dynamical variables $P$ and $Q$ (where,$_{a}=\partial / \partial a$ ) obtained from the variation of [188]

$$
\begin{aligned}
H= & \frac{1}{2} \int_{0}^{2 \pi} d \theta\left[\pi_{P}^{2}+e^{-2 P} \pi_{Q}^{2}\right] \\
& +\frac{1}{2} \int_{0}^{2 \pi} d \theta\left[e^{-2 \tau}\left(P_{,}^{2}+e^{2 P} Q,{ }_{\theta}^{2}\right)\right]=H_{1}+H_{2},
\end{aligned}
$$

where $\pi_{P}$ and $\pi_{Q}$ are canonically conjugate to $P$ and $Q$ respectively. This Hamiltonian has the form required by the symplectic scheme. If the model is, in fact, AVTD, the approximation in the symplectic numerical scheme should become more accurate as the singularity is approached. The second group of Einstein equations contains the Hamiltonian and $\theta$-momentum constraints, respectively. These can be expressed as first order equations for $\lambda$ in terms of $P$ and $Q$. This break into dynamical and constraint equations removes two of the most problematical areas of numerical relativity from this model - the initial value problem and numerical preservation of the constraints.

For the special case of the polarized Gowdy model $(Q=0), P$ satisfies a linear wave equation whose exact solution is well-known [26]. For this case, it has been proven that the singularity is AVTD [165]. This has also been conjectured to be true for generic Gowdy models [II2:3].

AVTD behavior is defined in [165] as follows: Solve the Gowdy wave equations neglecting all terms containing spatial derivatives. This yields the VTD

Living Reviews in Relativity (2002-1)

http://www.livingreviews.org 
solution [42]. If the approach to the singularity is AVTD, the full solution comes arbitrarily close to a VTD solution at each spatial point as $\tau \rightarrow \infty$. As $\tau \rightarrow \infty$, the VTD solution becomes

$$
P(\theta, \tau) \rightarrow v(\theta) \tau, \quad Q(\theta, \tau) \rightarrow Q_{0}(\theta)
$$

where $v>0$. Substitution in the wave equations shows that this behavior is consistent with asymptotic exponential decay of all terms containing spatial derivatives only if $0 \leq v<1$ [12:3]. We have shown that, except at isolated spatial points, the nonlinear terms in the wave equation for $P$ drive $v$ into this range [3.5, 37]. The exceptional points occur when coefficients of the nonlinear terms vanish and are responsible for the growth of spiky features seen in the wave forms [42, 35]. We conclude that generic Gowdy cosmologies have an AVTD singularity except at isolated spatial points [35, 37]. This has been confirmed by Hern and Stuart [14.3] and by van Putten [24.3]. After the nature of the solutions became clear through numerical experiments, it became possible to use Fuchsian asymptotic methods to prove that Gowdy solutions with $0<v<1$ and AVTD behavior almost everywhere are generic [173]. These methods have recently been applied to Gowdy spacetimes with $S^{2} \times S^{1}$ and $S^{3}$ topologies with similar conclusions [233].

One striking property of the Gowdy models are the development of "spiky features" at isolated spatial points where the coefficient of a local "potential term" vanishes [42, 3.5]. Recently, Rendall and Weaver have shown analytically how to generate such spikes from a Gowdy solution without spikes [220]].

Addition of a magnetic field to the vacuum Gowdy models (plus a topology change) which yields the inhomogeneous generalization of magnetic Bianchi $\mathrm{VI}_{0}$ models provides an additional potential which grows exponentially if $0<v<1$. Local Mixmaster behavior has recently been observed in these magnetic Gowdy models [2.50, 247].

Garfinkle has used a vacuum Gowdy model with $S^{2} \times S^{1}$ spatial topology to test an algorithm for axis regularity [II]]. Along the way, he has shown that these models are also AVTD with behavior at generic spatial points that is eventually identical to that in the $T^{3}$ case. Comparison of the two models illustrates that topology or other global or boundary conditions are important early in the simulation but become irrelevant as the singularity is approached.

Gowdy spacetimes are not the most general $T^{2}$ symmetric vacuum cosmologies. Certain off-diagonal metric components (the twists which are $g_{\theta \sigma}, g_{\theta \delta}$ in the notation of (12)) have been set to zero [12I]. Restoring these terms (see [8.3, 40] ) yields spacetimes that are not AVTD but rather appear to exhibit a novel type of LMD [4I, 249]. The LMD in these models is an inhomogeneous generalization of non-diagonal Bianchi models with "centrifugal" MSS potential walls [227, 169] in addition to the usual curvature walls. In [41], remarkable quantitative agreement is found between predictions of the MCP and numerical simulation of the full Einstein equations. A version of the code with AMR has been developed [15]. (Asymptotic methods have been used to prove that the polarized version of these spacetimes have AVTD solutions [163].)

Living Reviews in Relativity (2002-1)

http://www.livingreviews.org 


\subsubsection{U(1) symmetric cosmologies}

Moncrief has shown [190] that cosmological models on $T^{3} \times R$ with a spatial $U(1)$ symmetry can be described by five degrees of freedom $\{x, z, \Lambda, \varphi, \omega\}$ and their respective conjugate momenta $\left\{p_{x}, p_{z}, p_{\Lambda}, p, r\right\}$. All variables are functions of spatial variables $u, v$ and time $\tau$. Einstein's equations can be obtained by variation of

$$
\begin{aligned}
& H=\oint \oint d u d v \mathcal{H} \\
& =\oint \oint d u d v\left(\frac{1}{8} p_{z}^{2}+\frac{1}{2} e^{4 z} p_{x}^{2}+\frac{1}{8} p^{2}+\frac{1}{2} e^{4 \varphi} r^{2}-\frac{1}{2} p_{\Lambda}^{2}+2 p_{\Lambda}\right) \\
& +e^{-2 \tau} \oint \oint d u d v\left\{\left(e^{\Lambda} e^{a b}\right),{ }_{a b}-\left(e^{\Lambda} e^{a b}\right),{ }_{a} \Lambda,{ }_{b}\right. \\
& +e^{\Lambda}\left[\left(e^{-2 z}\right){ }_{u} x,,_{v}-\left(e^{-2 z}\right),{ }_{v} x,{ }_{u}\right] \\
& \left.+2 e^{\Lambda} e^{a b} \varphi,_{a} \varphi, b+\frac{1}{2} e^{\Lambda} e^{-4 \varphi} e^{a b} \omega,_{a} \omega, b\right\} \\
& =H_{1}+H_{2} .
\end{aligned}
$$

Here $\varphi$ and $\omega$ are analogous to $P$ and $Q$ while $e^{\Lambda}$ is a conformal factor for the metric $e_{a b}(x, z)$ in the $u-v$ plane perpendicular to the symmetry direction. Symplectic methods are still easily applicable. Note particularly that $H_{1}$ contains two copies of the Gowdy $H_{1}$ plus a free particle term and is thus exactly solvable. The potential term $H_{2}$ is very complicated. However, it still contains no momenta so its equations are trivially exactly solvable. However, issues of spatial differencing are problematic. (Currently, a scheme due to Norton [201] is used. A spectral evaluation of derivatives [100] which has been shown to work in Gowdy simulations [2:3] does not appear to be helpful in the $U(1)$ case.) A particular solution to the initial value problem is used since the general solution is not available [37].

Current limitations of the $U(1)$ code do not affect simulations for the polarized case since problematic spiky features do not develop. Polarized models have $r=\omega=0$. Grubišić and Moncrief [124] have conjectured that these polarized models are AVTD. The numerical simulations provide strong support for this conjecture [37, 44]. Asymptotic methods have been used to prove that an open set of AVTD solutions exist for this case [164].

\subsubsection{Going further}

The MCP indicates that the term containing gradients of $\omega$ in (14) acts as a Mixmaster-like potential to drive the system away from AVTD behavior in generic $U(1)$ models [24]. Numerical simulations provide support for this suggestion [37, 43]. Whether this potential term grows or decays depends on a function of the field momenta. This in turn is restricted by the Hamiltonian constraint.

Living Reviews in Relativity (2002-1)

http://www.livingreviews.org 


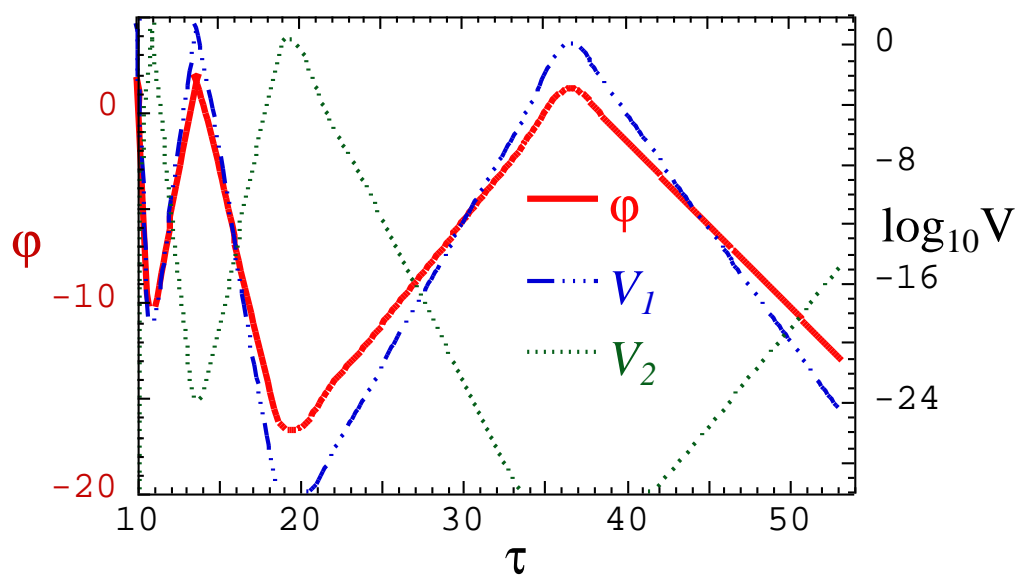

Figure 6: Behavior of the gravitational wave amplitude at a typical spatial point in a collapsing $U(1)$ symmetric cosmology. For details see [43, [36].

However, failure to enforce the constraints can cause an erroneous relationship among the momenta to yield qualitatively wrong behavior. There is numerical evidence that this error tends to suppress Mixmaster-like behavior leading to apparent AVTD behavior in extended spatial regions of $U(1)$ symmetric cosmologies [30, 3I]. In fact, it has been found [43], that when the Hamiltonian constraint is enforced at every time step, the predicted local oscillatory behavior of the approach to the singularity is observed. (The momentum constraint is not enforced.) (Note that in a numerical study of vacuum Bianchi IX homogeneous cosmologies, Zardecki obtained a spurious enhancement of Mixmaster oscillations due to constraint violation [25.3, 147]. In this case, the constraint violation introduced negative energy.)

Mixmaster simulations with the new algorithm [38] can easily evolve more than 250 bounces reaching $|\Omega| \approx 10^{62}$. This compares to earlier simulations yielding 30 or so bounces with $|\Omega| \approx 10^{8}$. The larger number of bounces quickly reveals that it is necessary to enforce the Hamiltonian constraint. An explicitly constraint enforcing $U(1)$ code was developed some years ago by Ove (see [2i17] and references therein).

It is well known [201] that a scalar field can suppress Mixmaster oscillations in homogeneous cosmologies. BKL argued that the suppression would also occur in spatially inhomogeneous models. This was demonstrated numerically for magnetic Gowdy and $U(1)$ symmetric spacetimes [33]. Andersson and Rendall proved that completely general cosmological (spatially $T^{3}$ ) spacetimes (no symmetries) with sufficiently strong scalar fields have generic AVTD solutions [3]. Garfinkle [107] has constructed a 3D harmonic code which, so far, has found AVTD solutions with a scalar field present. Work on generic vacuum models is in progress.

Cosmological models inspired by string theory contain higher derivative cur-

Living Reviews in Relativity (2002-1)

http://www.livingreviews.org 
vature terms and exotic matter fields. Damour and Henneaux have applied the BKL approach to such models and conclude that their approach to the singularity exhibits LMD [ 88$]$.

Finally, there has been a study of the relationship between the "long wavelength approximation" and the BKL analyses by Deruelle and Langlois [90].

Living Reviews in Relativity (2002-1)

http://www.livingreviews.org 


\section{Discussion}

Numerical investigation of singularities provides an arena for the close coupling of analytic and numerical techniques. The references provided here contain many examples of analytic results guided by numerical results and numerical studies to demonstrate whether or not analytic conjectures are valid.

Even more striking is the convergence of the separate topics of this review. While the search for naked singularities in the collapse of highly prolate systems has yielded controversial results, a naked singularity was discovered in the collapse of spherically symmetric scalar fields. The numerical exploration of cosmological singularities has yielded strong evidence that the asymptotic behavior is local - each spatial point evolves toward the singularity as a separate universe. This means that conclusions from these studies should be relevant in any generic collapse. This area of research then should begin to overlap with the studies of black hole interiors (see for example [64]).

Living Reviews in Relativity (2002-1)

http://www.livingreviews.org 


\section{Acknowledgements}

I would like to thank David Garfinkle for useful discussions (although any errors are mine). This update was supported in part by National Science Foundation Grant PHY9800103 to Oakland University.

Living Reviews in Relativity (2002-1)

http://www.livingreviews.org 


\section{References}

[1] Abrahams, A. M., and Evans, C. R., "Critical Behavior and Scaling in Vacuum Axisymmetric Gravitational Collapse", Phys. Rev. Lett., 70, 2980-2983, (1993). 2.2.1, 2.2.4

[2] Abrahams, A. M., Heiderich, K. R., Shapiro, S. L., and Teukolsky, S. A., "Vacuum Initial Data, Singularities, and Cosmic Censorship", Phys. Rev. $D, 46,2452-2463,(1992)$. 2.1 .3

[3] Andersson, L., and Rendall, A. D., "Quiescent Cosmological Singularity", Commun. Math. Phys., 218, 479-511, (2001). For a related online version see: L. Andersson, et al., "Quiescent Cosmological Singularity", (January, 2000), [Online Los Alamos Archive Preprint]: cited on 17 Jan 2000, http: //xxx.lanl.gov/abs/gr-qc/0001047. [, [3.4.4

[4] Anninos, P., "Computational Cosmology: from the Early Universe to the Large Scale Structure", Living Rev. Relativity, 4, 2001-2anninos, (February, 2001), [Online Journal Article]: cited on 2 Dec 2001, http: //www.livingreviews.org/Articles/Volume4/2001-2anninos/. 3.1

[5] Anninos, P., Centrella, J., and Matzner, R. A., "Nonlinear Wave Solutions to the Planar Vacuum Einstein Equations", Phys. Rev. D, 43, 1825-1838, (1991). 3.4.1

[6] Anninos, P., Centrella, J., and Matzner, R. A., "Numerical Methods for Solving the Planar Vacuum Einstein Equations", Phys. Rev. D, 43, 18081824, (1991). 3.4.1

[7] Ashtekar, A., Beetle, C., Dreyer, O., Fairhurs, S., Krishnan, B., Lewandowski, J., and Wisniewski, J., "Generic Isolated Horizons and Their Applications", Phys. Rev. Lett., 85, 3564-3567, (2000). For a related online version see: A. Ashtekar, et al., "Generic Isolated Horizons and Their Applications", (June, 2000), [Online Los Alamos Archive Preprint]: cited on 17 Aug 2000, http://xxx.lanl.gov/abs/gr-qc/0006006. [.].]

[8] Bañados, M., Teitelboim, C., and Zanelli, J., "Black Hole in ThreeDimensional Spacetime", Phys. Rev. Lett., 69, 1849-1851, (1992). 2.2.4

[9] Barrabès, C., Gremain, A., Lesigne, E., and Letelier, P. S., "Geometric Inequalities and the Hoop Conjecture", Class. Quantum Grav., 9, L105L110, (1992). 2.1.2

[10] Barrabès, C., Israel, W., and Letelier, P. S., "Analytic Models of Nonspherical Collapse, Cosmic Censorship and the Hoop Conjecture", Phys. Lett. A, 160, 41-44, (1991). 2.1.2, 2.1.3

[11] Barrow, J. D., "Chaotic Behaviour in General Relativity", Phys. Rep., 85, 1-49, (1982). 3.3.1

Living Reviews in Relativity (2002-1)

http://www.livingreviews.org 
[12] Barrow, J. D., and Levin, J., "Chaos in the Einstein-Yang-Mills Equations", Phys. Rev. Lett., 80, 656-659, (1998). For a related online version see: J. D. Barrow, et al., "Chaos in the Einstein-Yang-Mills Equations", (June, 1997), [Online Los Alamos Archive Preprint]: cited on 20 Jun 1997, http://xxx.lanl.gov/abs/gr-qc/9706065. 3.3.3

[13] Barrow, J. D., and Tipler, F., "Analysis of the Generic Singularity Studies by Belinskii, Khalatnikov, and Lifshitz", Phys. Rep., 56, 371-402, (1979). प, 3.4.1]

[14] Bartnik, R., and McKinnon, J., "Particle-like Solutions of the EinsteinYang-Mills Equations", Phys. Rev. Lett., 61, 141-144, (1988). 2.2.3

[15] Belanger, Z. B., Adaptive Mesh Refinement in the $T^{2}$ Symmetric Spacetime, Masters Thesis, (Oakland University, Rochester, 2001). 3.4 .2

[16] Belinskii, V. A., "Turbulence of a Gravitational Field near a Cosmological Singularity", JETP Lett., 56, 421-425, (1992). 3.4.1

[17] Belinskii, V. A., and Khalatnikov, I. M., "General Solution of the Gravitational Equations with a Physical Singularity", Sov. Phys. JETP, 30, 1174-1180, (1969). 1 1

[18] Belinskii, V. A., and Khalatnikov, I. M., "On the Nature of the Singularities in the General Solution of the Gravitational Equations", Sov. Phys. JETP, 29, 911-917, (1969). [1

[19] Belinskii, V. A., and Khalatnikov, I. M., "General Solution of the Gravitational Equations with a Physical Oscillatory Singularity", Sov. Phys. JETP, 32, 169-172, (1971). 1, 2.3.2

[20] Belinskii, V. A., and Khalatnikov, I. M., "Effect of Scalar and Vector Fields on the Nature of the Cosmological Singularity", Sov. Phys. JETP, 36, 591-597, (1973). 3.3.3, 3.4.4

[21] Belinskii, V. A., Khalatnikov, I. M., and Lifshitz, E. M., "A General Solution of the Einstein Equations with a Time Singularity", Adv. Phys., 31, 639-667, (1982). 1

[22] Belinskii, V. A., Lifshitz, E. M., and Khalatnikov, I. M., "Oscillatory Approach to the Singular Point in Relativistic Cosmology", Sov. Phys. Usp., 13, 745-765, (1971). [1, 3.1, 3.3.1, 3.4.1

[23] Berger, B. K., "A Spectral Symplectic Method for Numerical Investigation of Cosmological Singularities", (January, 2000), [Online Talk]: cited on 20 Jan 2000, http://online.itp.ucsb.edu/online/numrel00/berger/. 3.4 .3

[24] Berger, B. K., "On the Nature of the Generic Big Bang", (January, 1998), [Online Los Alamos Archive Preprint]: cited on 6 Jan 1998, http://xxx. lanl.gov/abs/gr-qc/9801010. 3.4.4

Living Reviews in Relativity (2002-1) http://www.livingreviews.org 
[25] Berger, B. K., "Numerical Approaches to Spacetime Singularities", Living Rev. Relativity, 1, 1998-7berger, (May, 1998), [Online Journal Article]: cited on 3 May 1998, http://www.livingreviews.org/Articles/ Volume1/1998-7berger. (document)

[26] Berger, B. K., "Quantum Graviton Creation in a Model Universe", Ann. Phys. (N. Y.), 83, 458-490, (1974). 3.4.1, 3.4.2

[27] Berger, B. K., "Comments on the Computation of Liapunov Exponents for the Mixmaster Universe", Gen. Relativ. Gravit., 23, 1385-1402, (1991). 3.3.

[28] Berger, B. K., "How to Determine Approximate Mixmaster Parameters from Numerical Evolution of Einstein's Equations", Phys. Rev. D, 49, 1120-1123, (1994). For a related online version see: B. K. Berger, "How to Determine Approximate Mixmaster Parameters from Numerical Evolution of Einstein's Equations", (August, 1993), [Online Los Alamos Archive Preprint]: cited on 17 Aug 1993, http://xxx.lanl.gov/abs/gr-qc/ 9308016. 凹, 3.3.1

[29] Berger, B. K., "Comment on the 'Chaotic' Singularity in Some Magnetic Bianchi $\mathrm{VI}_{0}$ Cosmologies", Class. Quantum Grav., 13, 1273-1276, (1996). For a related online version see: B. K. Berger, "Comment on the 'Chaotic' Singularity in Some Magnetic Bianchi $\mathrm{VI}_{0}$ Cosmologies", (December, 1995), [Online Los Alamos Archive Preprint]: cited on 1 Dec 1995, http://xxx.lanl.gov/abs/gr-qc/9512005. 3.1, 3.3.1

[30] Berger, B. K., "Numerical Investigation of Cosmological Singularities", in Hehl, F. W., Puntigam, R. A., and Ruder, H., eds., Relativity and Scientific Computing, 152-169, (Springer, Berlin, 1996). For a related online version see: B. K. Berger, "Numerical Investigation of Cosmological Singularities", (December, 1995), [Online Los Alamos Archive Preprint]: cited on 1 Dec 1995, http://xxx.lanl.gov/abs/gr-qc/9512004. 3.2.1, 3.4 .4

[31] Berger, B. K., "Numerical Investigation of Singularities", in Francaviglia, M., Longhi, G., Lusanna, L., and Sorace, E., eds., General Relativity and Gravitation, 57-78, (World Scientific, Singapore, 1997). For a related online version see: B. K. Berger, "Numerical Investigation of Singularities", (December, 1995), [Online Los Alamos Archive Preprint]: cited on 1 Dec 1995, http://xxx.lanl.gov/abs/gr-qc/9512003. [document), 3.4.4

[32] Berger, B. K., "Approach to the Singularity in Spatially Inhomogeneous Cosmologies", in Weikard, R., and Weinstein, G., eds., Differential Equations and Mathematical Physics: Proceedings of an International Conference Held at the University of Alabama, (American Mathematical Society, Providence, 2000). For a related online version see: B. K. Berger, "Approach to the Singularity in Spatially Inhomogeneous Cosmologies",

Living Reviews in Relativity (2002-1)

http://www.livingreviews.org 
(June, 2001), [Online Los Alamos Archive Preprint]: cited on 4 Jun 2001, http://xxx.lanl.gov/abs/gr-qc/0106009. [1, 3.4.1

[33] Berger, B. K., "Influence of Scalar Fields on the Approach to the Singularity in Spatially Inhomogeneous Cosmologies", Phys. Rev. D, 61, 023508, (2000). For a related online version see: B. K. Berger, "Influence of Scalar Fields on the Approach to the Singularity in Spatially Inhomogeneous Cosmologies", (July, 1999), [Online Los Alamos Archive Preprint]: cited on 26 Jul 1999, http://xxx.lanl.gov/abs/gr-qc/9907083. 3.4.1, 3.4 .4

[34] Berger, B. K., Chruściel, P. T., and Moncrief, V., "On 'Asymptotically Flat' Space-Times with $G_{2}-$ Invariant Cauchy Surfaces", Ann. Phys. (N. Y.), 237, 322-354, (1995). For a related online version see: B. K. Berger, et al., "On 'Asymptotically Flat' Space-Times with $G_{2}$-Invariant Cauchy Surfaces", (April, 1994), [Online Los Alamos Archive Preprint]: cited on 6 Apr 1994, http://xxx.lanl.gov/abs/gr-qc/9404005. 2.1.2, 2.1 .4

[35] Berger, B. K., and Garfinkle, D., "Phenomenology of the Gowdy Model on $T^{3} \times R$ ", Phys. Rev. D, 57, 4767-4777, (1998). For a related online version see: B. K. Berger, et al., "Phenomenology of the Gowdy Model on $T^{3} \times R$ ", (October, 1997), [Online Los Alamos Archive Preprint]: cited

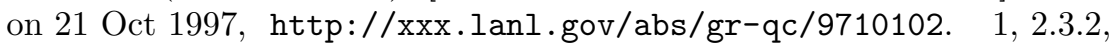
3.2.2, 3.4.1, 3.4.2

[36] Berger, B. K., Garfinkle, D., Isenberg, J., Moncrief, V., and Weaver, M., "The Singularity in Generic Gravitational Collapse is Spacelike, Local, and Oscillatory", Mod. Phys. Lett. A, 13, 1565-1573, (1998). For a related online version see: B. K. Berger, et al., "The Singularity in Generic Gravitational Collapse is Spacelike, Local, and Oscillatory", (May, 1998), [Online Los Alamos Archive Preprint]: cited on 17 May 1998, http://xxx.lanl.gov/abs/gr-qc/9805063. [1, 2.3.2, 3.4.1, 6

[37] Berger, B. K., Garfinkle, D., and Moncrief, V., "Numerical Study of Cosmological Singularities", in Burko, L. M., and Ori, A., eds., Internal Structure of Black Holes and Spacetime Singularities, 441-457, (Institute of Physics, Bristol, 1998). For a related online version see: B. K. Berger, et al., "Numerical Study of Cosmological Singularities", (September, 1997), [Online Los Alamos Archive Preprint]: cited on 26 Sep 1997, http://xxx.lanl.gov/abs/gr-qc/9709073. 3.2.1, 3.4.1, 3.4.2, 3.4.3, 3.4.3, 3.4.4

[38] Berger, B. K., Garfinkle, D., and Strasser, E., "New Algorithm for Mixmaster Dynamics", Class. Quantum Grav., 14, L29-L36, (1996). For a related online version see: B. K. Berger, et al., "New Algorithm for Mixmaster Dynamics", (September, 1997), [Online Los Alamos Archive Preprint]:

Living Reviews in Relativity (2002-1) http://www.livingreviews.org 
cited on 30 Sep 1996, http://xxx.lanl.gov/abs/gr-qc/9609072. 囵, 3.2.2, 5, 3.3.1, 3.3.2, 3.4.4

[39] Berger, B. K., Garfinkle, D., and Swamy, V., "Detection of Computer Generated Gravitational Waves in Numerical Cosmologies", Gen. Relativ. Gravit., 27, 511-527, (1995). For a related online version see: B. K. Berger, et al., "Detection of Computer Generated Gravitational Waves in Numerical Cosmologies", (May, 1994), [Online Los Alamos Archive Preprint]: cited on 27 May 1994, http://xxx.lanl.gov/abs/gr-qc/9405069. B.4.2

[40] Berger, B. K., Isenberg, J., Chruściel, P. T., and Moncrief, V., "Global Foliations of Vacuum Spacetimes with $T^{2}$ Isometry", Ann. Phys. (N. Y.), 260, 117-148, (1997). For a related online version see: B. K. Berger, et al., "Global Foliations of Vacuum Spacetimes with $T^{2}$ Isometry", (February, 1997), [Online Los Alamos Archive Preprint]: cited on 3 Feb 1997, http: //xxx.lanl.gov/abs/gr-qc/9702007. 3.4.1, 3.4.2

[41] Berger, B. K., Isenberg, J., and Weaver, M., "Oscillatory Approach to the Singularity in Vacuum Spacetimes with $T^{2}$ Isometry", Phys. Rev. D, 62, 123501, (2000). For a related online version see: B. K. Berger, et al., "Oscillatory Approach to the Singularity in Vacuum Spacetimes with $T^{2}$ Isometry", (April, 2001), [Online Los Alamos Archive Preprint]: cited on 16 Apr 2001, http://xxx.lanl.gov/abs/gr-qc/0104048. [1, 3.2.2, 3.4.1, 3.4.2

[42] Berger, B. K., and Moncrief, V., "Numerical Investigation of Cosmological Singularities", Phys. Rev. D, 48, 4676-4687, (1993). For a related online version see: B. K. Berger, et al., "Numerical Investigation of Cosmological Singularities", (July, 1993), [Online Los Alamos Archive Preprint]: cited on $22 \mathrm{Jul} 93$, http://xxx.lanl.gov/abs/gr-qc/9307032. [1, 3.2.1, 3.2.1, 3.2.2, 3.4.1, 3.4.2, 3.4.2

[43] Berger, B. K., and Moncrief, V., "Evidence for an Oscillatory Singularity in Generic $U(1)$ Symmetric Cosmologies on $T^{3} \times R$ ", Phys. Rev. D, 58, 064023, (1998). For a related online version see: B. K. Berger, et al., "Evidence for an Oscillatory Singularity in Generic $U(1)$ Symmetric Cosmologies on $T^{3} \times R^{\prime \prime}$, (April, 1998), [Online Los Alamos Archive Preprint]: cited on 30 Apr 1998, http://xxx.lanl.gov/abs/gr-qc/9804085. 1, 3.2.2, 3.4.1, 3.4.4, [6

[44] Berger, B. K., and Moncrief, V., "Numerical Evidence that the Singularity in Polarized $U(1)$ Symmetric Cosmologies on $T^{3} \times R$ is Velocity Dominated", Phys. Rev. D, 57, 7235-7240, (1998). For a related online version see: B. K. Berger, et al., "Numerical Evidence that the Singularity in Polarized $U(1)$ Symmetric Cosmologies on $T^{3} \times R$ is Velocity Dominated", (January, 1998), [Online Los Alamos Archive Preprint]: cited on 22 Jan 1998, http://xxx.lanl.gov/abs/gr-qc/9801078. [1, 3.4.3

Living Reviews in Relativity (2002-1)

http://www.livingreviews.org 
[45] Berger, B. K., and Moncrief, V., "Signature for Local Mixmaster Dynamics in $U(1)$ Symmetric Cosmologies", Phys. Rev. D, 62, 123501, (2000). For a related online version see: B. K. Berger, et al., "Signature for Local Mixmaster Dynamics in U(1) Symmetric Cosmologies", (June, 2000), [Online Los Alamos Archive Preprint]: cited on 20 Jun 2000, http://xxx.lanl.gov/abs/gr-qc/0006071. 3.4.1

[46] Bizoń, P., "How to Make a Tiny Black Hole?", Acta Cosmologica, 22, 81, (1996). For a related online version see: P. Bizoń, "How to Make a Tiny Black Hole?", (June, 1996), [Online Los Alamos Archive Preprint]: cited on 19 Jun 1996, http://xxx.lanl.gov/abs/gr-qc/9606060. [1

[47] Bizoń, P., and Chmaj, T., "Formation and Critical Collapse of Skyrmions", Phys. Rev. D, 58, 041501, (1998). For a related online version see: P. Bizoń, et al., "Critical Collapse of Skyrmions", (January, 1998), [Online Los Alamos Archive Preprint]: cited on 12 Jan 1998, http://xxx.lanl.gov/abs/gr-qc/9801012. 2.2.3

[48] Bizoń, P., Chmaj, T., and Tabor, Z., "Dispersion and Collapse of Wave Maps", Nonlinearity, 13, 1411-1423, (2000). For a related online version see: P. Bizon, et al., "Dispersion and Collapse of Wave Maps", (December, 1999), [Online Los Alamos Archive Preprint]: cited on 17 Dec 1999, http: //xxx.lanl.gov/abs/math-ph/9912009. 2.2.4

[49] Bizoń, P., and Tabor, Z., "On Blowup for Yang-Mills Fields", Phys. Rev. $D$, 64, 121701, (2001). For a related online version see: P. Bizoń, et al., "On Blowup for Yang-Mills Fields", (May, 2001), [Online Los Alamos Archive Preprint]: cited on 16 May 2001, http://xxx.lanl.gov/abs/ math-ph/0105016. 2.2.4

[50] Bonanno, A., Droz, S., Israel, W., and Morsink, S. M., "Structure of the Spherical Black Hole Interior", Proc. R. Soc. London, Ser. A, 450, 553-567, (1995). For a related online version see: A. Bonanno, et al., "Structure of the Spherical Black Hole Interior", (November, 1994), [Online Los Alamos Archive Preprint]: cited on 18 Nov 1994, http://xxx.lanl.gov/abs/gr-qc/9411050. 2.3.2

[51] Brady, P. R., and Chambers, C. M., "Non-Linear Instability of Kerr-Type Cauchy Horizons", Phys. Rev. D, 51, 4177-4186, (1995). For a related online version see: P. R. Brady, et al., "Non-Linear Instability of KerrType Cauchy Horizons", (January, 1995), [Online Los Alamos Archive Preprint]: cited on 21 Jan 1995, http://xxx.lanl.gov/abs/gr-qc/ 950102.5. ए32

[52] Brady, P. R., Chambers, C. M., and Gonçalves, S. M. C. V., "Phases of Massive Scalar Field Collapse", Phys. Rev. D, 56, 6057-6061, (1997). For a related online version see: P. R. Brady, et al., "Phases of Massive Scalar Field Collapse", (September, 1997), [Online Los Alamos Archive 
Preprint]: cited on 5 Sep 1997, http://xxx.lanl.gov/gr-qc/9709014. 2.2 .3

[53] Brady, P. R., Chambers, C. M., Krivan, W., and Laguna, P., "Telling Tails in the Presence of a Cosmological Constant", Phys. Rev. D, 55, 7538-7545, (1997). For a related online version see: P. R. Brady, et al., "Telling Tails in the Presence of a Cosmological Constant", (November, 1996), [Online Los Alamos Archive Preprint]: cited on 23 Nov 1996, http: //xxx.lanl.gov/abs/gr-qc/9611056. 2.3.3

[54] Brady, P. R., Droz, S., and Morsink, S. M., "Late-Time Singularity inside Nonspherical Black Holes", Phys. Rev. D, 58, 084034, (1998). For a related online version see: P. R. Brady, et al., "The Late-Time Singularity inside Nonspherical Black Holes", (May, 1998), [Online Los Alamos Archive Preprint]: cited on 5 May 1998, http://xxx.lanl.gov/abs/ gr-qc/9805008. 2.3.2

[55] Brady, P. R., Moss, I. G., and Myers, R. C., "Cosmic Censorship: As Strong as Ever", Phys. Rev. Lett., 80, 3432-3435, (1998). For a related online version see: P. R. Brady, et al., "Cosmic Censorship: As Strong as Ever", (January, 1998), [Online Los Alamos Archive Preprint]: cited on 12 Jan 1998, http://xxx.lanl.gov/abs/gr-qc/9801032. 2.3.3

[56] Brady, P. R., and Smith, J. D., "Black Hole Singularities a Numerical Approach", Phys. Rev. Lett., 75, 1256-1259, (1995). For a related online version see: P. R. Brady, et al., "Black Hole Singularities a Numerical Approach", (June, 1995), [Online Los Alamos Archive Preprint]: cited on 28 Jun 1995, http://xxx.lanl.gov/abs/gr-qc/950607. [1, 2.3.2, 2.3 .2 , 2.3.3

[57] Breitenlohner, P., Lavrelashvili, G., and Maison, D., "Mass Inflation and Chaotic Behavior Inside Hairy Black Holes", Nucl. Phys. B, 524, 427-443, (1998). For a related online version see: P. Breitenlohner, et al., "Mass Inflation and Chaotic Behavior Inside Hairy Black Holes", (March, 1997), [Online Los Alamos Archive Preprint]: cited on 18 Mar 1997, http: //xxx.lanl.gov/abs/gr-qc/9703047. 2.3.2

[58] Breitenlohner, P., Lavrelashvili, G., and Maison, D., "Non-Abelian Black Holes: The Inside Story", in Burko, L. M., and Ori, A., eds., Internal Structure of Black Holes and Spacetime Singularities, 172-193, (Institute of Physics, Bristol, 1998). For a related online version see: P. Breitenlohner, et al., "Non-Abelian Black Holes: The Inside Story", (August, 1997), [Online Los Alamos Archive Preprint]: cited on 17 Aug 1997, http://xxx.lanl.gov/abs/gr-qc/9708036. 2.3.2

[59] Breitenlohner, P., Lavrelashvili, G., and Maison, D., "Mass Inflation Inside Non-Abelian Black Holes", in Piran, T., ed., Proceedings of the

Living Reviews in Relativity (2002-1)

http://www.livingreviews.org 
Eighth Marcel Grossmann Meeting on General Relativity, (World Scientific, Singapore, 1999). For a related online version see: P. Breitenlohner, et al., "Mass Inflation Inside Non-Abelian Black Holes", (November, 1997), [Online Los Alamos Archive Preprint]: cited on 6 Nov 1997, http://xxx.lanl.gov/abs/gr-qc/9711024. 2.3.2

[60] Brill, D. R., "On the Positive Definite Mass of the Bondi-Weber-Wheeler Time-Symmetric Gravitational Waves", Ann. Phys. (N. Y.), 7, 466-483, (1959). 2.1 .3

[61] Browne, M. W., "A Bet on a Cosmic Scale, and a Concession Sort of", The New York Times, (12 Feb 1997). 2.2.1

[62] Burd, A. B., Buric, N., and Ellis, G. F. R., "A Numerical Analysis of Chaotic Behavior in Bianchi IX Models", Gen. Relativ. Gravit., 22, 349 363, (1990). 1, 3.3.1

[63] Burko, L. M., "Structure of the Black Hole's Cauchy Horizon Singularity", Phys. Rev. Lett., 79, 4958-4961, (1997). For a related online version see: L. M. Burko, "Structure of the Black Hole's Cauchy Horizon Singularity", (October, 1997), [Online Los Alamos Archive Preprint]: cited on 26 Oct 1997, http://xxx.lanl.gov/abs/gr-qc/9710112. [1, 2.3.2

[64] Burko, L. M., "Homogeneous Spacelike Singularities Inside Spherical Black Holes", in Burko, L. M., and Ori, A., eds., Internal Structure of Black Holes and Spacetime Singularities, (Institute of Physics, Bristol, 1998). For a related online version see: L. M. Burko, "Homogeneous Spacelike Singularities Inside Spherical Black Holes", (November, 1997), [Online Los Alamos Archive Preprint]: cited on 5 Nov 1997, http://xxx.lanl.gov/abs/gr-qc/9711012. 困

[65] Burko, L. M., "The Singularity in Supercritical Collapse of a Spherical Scalar Field", Phys. Rev. D, 58, 084013, (1998). For a related online version see: L. M. Burko, "The Singularity in Supercritical Collapse of a Spherical Scalar Field", (March, 1998), [Online Los Alamos Archive Preprint]: cited on 22 Mar 1998, http://xxx.lanl.gov/abs/gr-qc/ 9803059. 2.3.2

[66] Burko, L. M., "Singularity Deep inside the Charged Black Hole Core", Phys. Rev. D, 59, 024011, (1999). For a related online version see: L. M. Burko, "Singularity Deep inside the Charged Black Hole Core", (July, 1999), [Online Los Alamos Archive Preprint]: cited on 27 Sep 1998, http: //xxx.lanl.gov/abs/gr-qc/9809073. 困

[67] Burko, L. M., and Ori, A., "Late-Time Evolution of Nonlinear Gravitational Collapse", Phys. Rev. D, 56, 7820-7832, (1997). For a related online version see: L. M. Burko, et al., "Late-Time Evolution of Nonlinear Gravitational Collapse", (March, 1997), [Online Los Alamos Archive Preprint]: cited on 21 Sep 1997, http://xxx.lanl.gov/abs/gr-qc/9703067. 2.3.3 
[68] Burko, L. M., and Ori, A., "Analytic Study of the Null Singularity inside Spherical Charged Black Holes", Phys. Rev. D, 57, 7084-7088, (1998). For a related online version see: L. M. Burko, et al., "Analytic Study of the Null Singularity inside Spherical Charged Black Holes", (November, 1997), [Online Los Alamos Archive Preprint]: cited on 10 Nov 1997, http: //xxx.lanl.gov/abs/gr-qc/9711032. 2.3.2

[69] Carretero-Gonzalez, R., Nunuz-Yepez, H. N., and Salas-Brito, A. L., "Evidence of Chaotic Behavior in Jordan-Brans-Dicke Cosmology", Phys. Lett. A, 188, 48, (1994). 3.3 .3

[70] Chambers, C. M., "The Cauchy Horizon in Black Hole-de Sitter Spacetimes", in Burko, L. M., and Ori, A., eds., Internal Structure of Black Holes and Spacetime Singularities, (Institute of Physics, Bristol, 1998). For a related online version see: C. M. Chambers, "The Cauchy Horizon in Black Hole-de Sitter Spacetimes", (September, 1997), [Online Los Alamos Archive Preprint]: cited on 11 Sep 1997, http://xxx.lanl.gov/ abs/gr-qc/9709025. 2.3.3

[71] Chambers, C. M., Brady, P. R., and Gonçalves, S. M. C. V., "Phases of Massive Scalar Field Collapse", in Piran, T., ed., Proceedings of the Eighth Marcel Grossmann Meeting on General Relativity, (World Scientific, Singapore, 1999). For a related online version see: C. M. Chambers, et al., "Phases of Massive Scalar Field Collapse", (October, 1997), [Online Los Alamos Archive Preprint]: cited on 2 Oct 1997, http: //xxx.lanl.gov/gr-qc/9710014. 2.2.3

[72] Chambers, C. M., Brady, P. R., Krivan, W., and Laguna, P., "Some Cosmological Tails of Collapse", in Piran, T., ed., Proceedings of the Eighth Marcel Grossmann Meeting on General Relativity, (World Scientific, Singapore, 1999). For a related online version see: C. M. Chambers, et al., "Some Cosmological Tails of Collapse", (October, 1997), [Online Los Alamos Archive Preprint]: cited on 2 Oct 1997, http: //xxx.lanl.gov/abs/gr-qc/9710015. 2.3.3

[73] Chandrasekhar, S., and Hartle, J. B., "On Crossing the Cauchy Horizon of a Reissner-Nordström Black Hole", Proc. R. Soc. London, Ser. A, 384, 301-315, (1982). 1

[74] Chernoff, D. F., and Barrow, J. D., "Chaos in the Mixmaster Universe", Phys. Rev. Lett., 50, 134-137, (1983). 3.3.1

[75] Chiba, T., "Apparent Horizon Formation and Hoop Conjecture in Nonaxisymmetric Spaces", Phys. Rev. D, 60, 044003, (1999). For a related online version see: T. Chiba, "Apparent Horizon Formation and Hoop Conjecture in Non-axisymmetric Spaces", (April, 1999), [Online Los Alamos Archive Preprint]: cited on 4 Jul 99, http://xxx.lanl.gov/abs/gr-qc/ 9904054. 2.1.1, 2.1.4

Living Reviews in Relativity (2002-1) http://www.livingreviews.org 
[76] Chiba, T., Nakamura, T., Nakao, K., and Sasaki, M., "Hoop Conjecture for Apparent Horizon Formation", Class. Quantum Grav., 11, 431-441, (1994). 2.1.2, 2.1.4

[77] Choptuik, M. W., "Animations of SU(2) EYM Collapse", (1999), [Online HTML Document]: cited on 20 June 2001, http://laplace.physics. ubc.ca/People/matt/Movies/YM/index.html. [3]

[78] Choptuik, M. W., "Universality and Scaling in Gravitational Collapse of a Massive Scalar Field", Phys. Rev. Lett., 70, 9-12, (1993). 1, 2.2.1, 2.3 .3

[79] Choptuik, M. W., Chmaj, T., and Bizoń, P., "Critical Behavior in Gravitational Collapse of a Yang-Mills Field", Phys. Rev. Lett., 77, 424-427, (1996). For a related online version see: M. W. Choptuik, et al., "Critical Behavior in Gravitational Collapse of a Yang-Mills Field", (March, 1996), [Online Los Alamos Archive Preprint]: cited on 27 Mar 96, http://xxx.lanl.gov/abs/gr-qc/9603051. 2.2.3

[80] Christodoulou, D., "A Mathematical Theory of Gravitational Collapse", Commun. Math. Phys., 109, 613-647, (1987). 2.2.1

[81] Christodoulou, D., "The Instability of Naked Singularities in the Gravitational Collapse of a Scalar Field", Ann. Math., 149, 183-217, (1999). 四

[82] Chruściel, P. T., "On Space-Times with $U(1) \times U(1)$ Symmetrical Compact Cauchy Surfaces", Ann. Phys. (N. Y.), 202, 100-150, (1990). 3.4.1

[83] Chrusciel, P. T., Isenberg, J., and Moncrief, V., "Strong Cosmic Censorship in Polarised Gowdy Spacetimes", Ann. Phys. (N. Y.), 7, 1671-1680, (1990). 3.4 .2

[84] Coley, A., "No Chaos in Brane-World Cosmology", (October, 2001), [Online Los Alamos Archive Preprint]: cited on 12 Oct 2001, http: //xxx.lanl.gov/abs/hep-th/0110117. 3.3.3

[85] Cornish, N. J., and Levin, J., "The Mixmaster Universe: A Chaotic Farey Tale", Phys. Rev. D, 55, 7489-7510, (1997). For a related online version see: N. J. Cornish, et al., "The Mixmaster Universe: A Chaotic Farey Tale", (December, 1996), [Online Los Alamos Archive Preprint]: cited on 30 Dec 1996, http://xxx.lanl.gov/abs/gr-qc/9612066. 3.3.1

[86] Cornish, N. J., and Levin, J., "The Mixmaster Universe is Chaotic", Phys. Rev. Lett., 78, 998-1001, (1997). For a related online version see: N. J. Cornish, et al., "The Mixmaster Universe is Chaotic", (May, 1996), [Online Los Alamos Archive Preprint]: cited on 6 Nov 1996, http://xxx.lanl.gov/abs/gr-qc/9605029. [, 3.1, 3.3.1, 3.3.1] 
[87] Cotsakis, S., Demaret, J., DeRop, Y., and Querella, L., "Mixmaster Universe in Fourth-Order Gravity Theories", Phys. Rev. D, 48, 4595-4603, (1993). $\quad 3.3 .3$

[88] Damour, T., and Henneaux, M., "Chaos in Superstring Cosmology", Phys. Rev. Lett., 85, 920-923, (2000). For a related online version see: T. Damour, et al., "Chaos in Superstring Cosmology", (March, 2000), [Online Los Alamos Archive Preprint]: cited on 16 Mar 2000, http: //xxx.lanl.gov/abs/hep-th/0003139. 3.4.4

[89] Damour, T., and Henneaux, M., "Oscillatory Behaviour in Homogeneous String Cosmology Models", Phys. Lett. B, 488, 108-116, (2000). For a related online version see: T. Damour, et al., "Oscillatory Behaviour in Homogeneous String Cosmology Models", (June, 2000), [Online Los Alamos Archive Preprint]: cited on 21 Jun 2000, http://xxx.lanl.gov/abs/ hep-th/0006171. $\quad 3.3 .3$

[90] Deruelle, N., and Langlois, D., "Long Wavelength Iteration of Einstein's Equations near a Spacetime Singularity", Phys. Rev. D, 52, 2007-2019, (1995). For a related online version see: N. Deruelle, et al., "Long Wavelength Iteration of Einstein's Equations near a Spacetime Singularity", (November, 1994), [Online Los Alamos Archive Preprint]: cited on 15 Nov 1994, http://xxx.lanl.gov/abs/gr-qc/94110. 3.4.4

[91] Donets, E. E., Gal'tsov, D. V., and Zotov, M. Yu., "Internal Structure of Einstein-Yang-Mills Black Holes", Phys. Rev. D, 56, 3459-3465, (1997). For a related online version see: E. E. Donets, et al., "Internal Structure of Einstein-Yang-Mills Black Holes", (December, 1996), [Online Los Alamos Archive Preprint]: cited on 23 Apr 1997, http://xxx.lanl.gov/abs/ gr-qc/9612067. 2.3.2

[92] Droz, S., "Numerical Investigation of Black Hole Interiors", Helv. Phys. Acta, 69, 257-260, (1996). For a related online version see: S. Droz, "Numerical Investigation of Black Hole Interiors", (August, 1996), [Online Los Alamos Archive Preprint]: cited on 14 Aug 1996, http://xxx.lanl. gov/abs/gr-qc/9608034. [1, 2.3.2

[93] Eardley, D., Isenberg, J., Marsden, J., and Moncrief, V., "Homothetic and Conformal Symmetries of Solutions to Einstein's Equations", Commun. Math. Phys., 106, 137-158, (1986). 2.2 .2

[94] Eardley, D., Liang, E., and Sachs, R., "Velocity-Dominated Singularities in Irrotational Dust Cosmologies", J. Math. Phys., 13, 99-107, (1972). 田, 3.1

[95] Eardley, D. M., Hirschmann, E. W., and Horne, J. H., "S-Duality at the Black Hole Threshold in Gravitational Collapse", Phys. Rev. D, 52, 5397-5401, (1995). For a related online version see: D. M. Eardley, et al., "S-Duality at the Black Hole Threshold in Gravitational Collapse",

Living Reviews in Relativity (2002-1)

http://www.livingreviews.org 
(May, 1995), [Online Los Alamos Archive Preprint]: cited on 22 May 1995, http://xxx.lanl.gov/abs/gr-qc/9505041. 2.2.3

[96] Echeverria, F., "Gravitational Collapse of an Infinite, Cylindrical Dust Shell", Phys. Rev. D, 47, 2271-2282, (1993). 2.1.4

[97] Ellis, G. F. R., and Schmidt, B. G., "Singular Space-Times", Gen. Relativ. Gravit., 8, 915-953, (1977). 1

[98] Evans, C. R., and Coleman, J. S., "Critical Phenomena and Self-Similarity in the Gravitational Collapse of Radiation Fluid", Phys. Rev. Lett., 72, 1782-1785, (1994). For a related online version see: C. R. Evans, et al., "Observation of Critical Phenomena and Self-Similarity in the Gravitational Collapse of Radiation Fluid", (February, 1994), [Online Los Alamos Archive Preprint]: cited on 22 Feb 1994, http://xxx.lanl.gov/abs/ gr-qc/9402041. 2.2.2

[99] Ferraz, K., Francisco, G., and Matsas, G. E. A., "Chaotic and Nonchaotic Behavior in the Mixmaster Dynamics", Phys. Lett. A, 156, 407, (1991). B.3.

[100] Finn, L. S., "A Numerical Approach to Binary Black Hole Coalescence", in Francaviglia, M., Longhi, G., Lusanna, L., and Sorace, E., eds., General Relativity and Gravitation, 147-166, (World Scientific, Singapore, 1997). For a related online version see: L. S. Finn, "A Numerical Approach to Binary Black Hole Coalescence", (March, 1996), [Online Los Alamos Archive Preprint]: cited on 5 Mar 1996, http://xxx.lanl.gov/abs/ gr-qc/9603004. [1, 3.4.3

[101] Fleck, J. A., Morris, J. R., and Feit, M. D., "Time-Dependent Propagation of High Energy Laser Beams through the Atmosphere", Appl. Phys., 10, 129-160, (1976). 3.2.1

[102] Francisco, G., and Matsas, G. E. A., "Qualitative and Numerical Study of Bianchi IX Models", Gen. Relativ. Gravit., 20, 1047-1054, (1988). 1, 5.3.

[103] Friedrich, H., "On Static and Radiative Spacetimes", Commun. Math. Phys., 119, 51-73, (1988). 2.3 .3

[104] Gal'tsov, D. V., and Donets, E. E., "Power-Law Mass Inflation in Einstein-Yang-Mills-Higgs Black Holes", (June, 1997), [Online Los Alamos Archive Preprint]: cited on 22 Jun 1997, http://xxx.lanl. gov/abs/gr-qc/9706067. 2.3.2

[105] Gal'tsov, D. V., Donets, E. E., and Zotov, M. Yu., "Singularities Inside Non-Abelian Black Holes", JETP Lett., 65, 895-901, (1997). For a related online version see: D. V. Gal'tsov, et al., "Singularities Inside Non-Abelian Black Holes", (June, 1997), [Online Los Alamos Archive Preprint]: cited on 20 Jun 1997, http://xxx.lanl.gov/abs/gr-qc/9706063. 2.3.2

Living Reviews in Relativity (2002-1) http://www.livingreviews.org 
[106] Gal'tsov, D. V., Donets, E. E., and Zotov, M. Yu., "Singularities inside Hairy Black Holes", in Piran, T., ed., Proceedings of the Eighth Marcel Grossmann Meeting on General Relativity, (World Scientific, Singapore, 1999). For a related online version see: D. V. Gal'tsov, et al., "Singularities inside Hairy Black Holes", (December, 1997), [Online Los Alamos Archive Preprint]: cited on 1 Dec 1997, http://xxx.lanl.gov/abs/ gr-qc/9712003. 2.3.2

[107] Garfinkle, D., "Harmonic Coordinate Method for Simulating Generic Singularities", (October, 2001), [Online Los Alamos Archive Preprint]: cited on 1 Oct 2001, http://xxx.lanl.gov/abs/gr-qc/0110013. 3.4.4

[108] Garfinkle, D., "Asymptotically Flat Space-Times Have No Conformal Killing Fields", J. Math. Phys., 28, 28-32, (1987). 2.2 .2

[109] Garfinkle, D., "Choptuik Scaling in Null Coordinates", Phys. Rev. D, 51, 5558-5561, (1995). For a related online version see: D. Garfinkle, "Choptuik Scaling in Null Coordinates", (December, 1994), [Online Los Alamos Archive Preprint]: cited on 1 Dec 1994, http://xxx.lanl.gov/ abs/gr-qc/9412008. 2.2.1

[110] Garfinkle, D., "Choptuik Scaling and the Scale Invariance of Einstein's Equation", Phys. Rev. D, 56, 3169-3173, (1997). For a related online version see: D. Garfinkle, "Choptuik Scaling and the Scale Invariance of Einstein's Equation", (December, 1996), [Online Los Alamos Archive Preprint]: cited on 31 Jul 1997, http://xxx.lanl.gov/abs/gr-qc/ 9612015. 2.2.4

[111] Garfinkle, D., "Numerical Simulations of Gowdy Spacetimes on $S^{2} \times S^{1} \times$ R", Phys. Rev. D, 60, 104010, (1999). For a related online version see: D. Garfinkle, "Numerical Simulations of Gowdy Spacetimes on $S^{2} \times S^{1} \times$ R", (June, 1999), [Online Los Alamos Archive Preprint]: cited on 4 Jun 1999, http://xxx.lanl.gov/abs/gr-qc/9906019. 2.3.2, 3.2.2, 3.4.2

[112] Garfinkle, D., "Exact Solution for 2+1-Dimensional Critical Collapse", Phys. Rev. D, 63, 044007, (2001). For a related online version see: D. Garfinkle, "An Exact Solution for 2+1 Dimensional Critical Collapse", (August, 2000), [Online Los Alamos Archive Preprint]: cited on 12 Nov 2000, http://xxx.lanl.gov/abs/gr-qc/0008023. 2.2.4

[113] Garfinkle, D., and Duncan, G. C., "Scaling of Curvature in Sub-Critical Gravitational Collapse", Phys. Rev. D, 58, 064024, (1998). For a related online version see: D. Garfinkle, et al., "Scaling of Curvature in SubCritical Gravitational Collapse", (February, 1998), [Online Los Alamos Archive Preprint]: cited on 24 Feb 1998, http://xxx.lanl.gov/abs/ gr-qc/9802061. 2.2.3

[114] Garfinkle, D., and Duncan, G. C., "Numerical Evolution of Brill Waves", Phys. Rev. D, 63, 044011, (2001). For a related online version see:

Living Reviews in Relativity (2002-1)

http://www.livingreviews.org 
D. Garfinkle, et al., "Numerical Evolution of Brill Waves", (June, 2000), [Online Los Alamos Archive Preprint]: cited on 20 Oct 2000, http: //xxx.lanl.gov/abs/gr-qc/0006073. 2.1.3

[115] Garfinkle, D., and Gundlach, C., "Symmetry-Seeking Spacetime Coordinates", Class. Quantum Grav., 16, 4111-4123, (1999). For a related online version see: D. Garfinkle, et al., "Symmetry-Seeking Spacetime Coordinates", (August, 1999), [Online Los Alamos Archive Preprint]: cited on 28 Oct 1999, http://xxx.lanl.gov/abs/gr-qc/9908016. 2.2.4

[116] Garfinkle, D., and Meyer, K., "Scale Invariance and Critical Gravitational Collapse", Phys. Rev. D, 59, 0640003, (1999). For a related online version see: D. Garfinkle, et al., "Scale Invariance and Critical Gravitational Collapse", (June, 1998), [Online Los Alamos Archive Preprint]: cited on 12 Aug 1998, http://xxx.lanl.gov/abs/gr-qc/9806052. 2.2.4

[117] Gentle, A. P., and Miller, W. A., "A Fully 3+1-D Regge Calculus Model of the Kasner Cosmology", Class. Quantum Grav., 15, 389-405, (1998). For a related online version see: A. P. Gentle, et al., "A Fully $3+1-D$ Regge Calculus Model of the Kasner Cosmology", (June, 1997), [Online Los Alamos Archive Preprint]: cited on 12 Jun 1997, http://xxx.lanl. gov/abs/gr-qc/9706034. 3.3 .3

[118] Gnedin, M. L., and Gnedin, N. Y., "Destruction of the Cauchy Horizon in the Reissner-Nordström Black Hole", Class. Quantum Grav., 10, 10831102, (1993). 2.3 .2

[119] Goldwirth, D. S., and Piran, T., "Gravitational Collapse of Massless Scalar Field and Cosmic Censorship", Phys. Rev. D, 36, 3575-3581, (1987). 2.2.1, 2.3.2

[120] Gonçalves, S. M. C. V., and Moss, I. G., "Black Hole Formation from Massive Scalar Fields", Class. Quantum Grav., 14, 2607-2615, (1997). For a related online version see: S. M. C. V. Gonçalves, et al., "Black Hole Formation from Massive Scalar Fields", (February, 1997), [Online Los Alamos Archive Preprint]: cited on 27 Feb 1997, http://xxx.lanl. gov/abs/gr-qc/9702059. 2.2.3

[121] Gowdy, R. H., "Gravitational Waves in Closed Universes", Phys. Rev. Lett., 27, 826, (1971). 2.3.2, 3.4.1, 3.4.2

[122] Grubišić, B., "Velocity Dominance near a Crushing Singularity", in Brown, J. D., Chu, M. T., Ellison, D. C., and Plemmons, R. J., eds., Proceedings of the Cornelius Lanczos Symposium, (SIAM, Philadelphia, 1994). For a related online version see: B. Grubišić, "Velocity Dominance near a Crushing Singularity", (April, 1994), [Online Los Alamos Archive Preprint]: cited on 27 Apr 1994, http://xxx.lanl.gov/abs/gr-qc/ 9404056. 3.4. 
[123] Grubišić, B., and Moncrief, V., "Asymptotic Behavior of the $T^{3} \times R$ Gowdy Space-times", Phys. Rev. D, 47, 2371-2382, (1993). For a related online version see: B. Grubišić, et al., "Asymptotic Behavior of the $T^{3} \times R$ Gowdy Space-times", (September, 1992), [Online Los Alamos Archive Preprint]: cited on 15 Sep 1994, http://xxx.lanl.gov/abs/ gr-qc/9209006. [1, 3.4.1, 3.4.2, 3.4.2

[124] Grubišić, B., and Moncrief, V., "Mixmaster Spacetime, Geroch's Transformation, and Constants of Motion", Phys. Rev. D, 49, 2792-2800, (1994). For a related online version see: B. Grubišić, et al., "Mixmaster Spacetime, Geroch's Transformation, and Constants of Motion", (September, 1993), [Online Los Alamos Archive Preprint]: cited on 9 Sep 1993, http://xxx.lanl.gov/abs/gr-qc/9309007. 3.4.3

[125] Gundlach, C., "Critical Phenomena in Gravitational Collapse", (June, 1996), [Online Los Alamos Archive Preprint]: cited on 12 Jun 1996, http://xxx.lanl.gov/ps/gr-qc/9606023. Submitted to Banach Centre Proceedings. [1, 2.2.1

[126] Gundlach, C., "Critical Phenomena in Gravitational Collapse", Living Rev. Relativity, 2, 1999-4gundlach, (December, 1999), [Online Journal Article]: cited on 28 May 2001, http://www.livingreviews.org/ Articles/Volume2/1999-4gundlach. [1, 2.2.1, 2.2.2

[127] Gundlach, C., "The Choptuik Spacetime as an Eigenvalue Problem", Phys. Rev. Lett., 75, 3214-3217, (1995). For a related online version see: C. Gundlach, "The Choptuik Spacetime as an Eigenvalue Problem", (July, 1995), [Online Los Alamos Archive Preprint]: cited on 13 Sep 1995, http://xxx.lanl.gov/abs/gr-qc/9507054. 2.2.2

[128] Gundlach, C., "Echoing and Scaling in Einstein-Yang-Mills Critical Collapse", Phys. Rev. D, 55, 6002-6013, (1997). For a related online version see: C. Gundlach, "Echoing and Scaling in Einstein-Yang-Mills Critical Collapse", (October, 1996), [Online Los Alamos Archive Preprint]: cited on 4 Nov 1996, http://xxx.lanl.gov/abs/gr-qc/9610069. 2.2.3

[129] Gundlach, C., "Understanding Critical Collapse of a Scalar Field", Phys. Rev. D, 55, 695-713, (1997). For a related online version see: C. Gundlach, "Understanding Critical Collapse of a Scalar Field", (April, 1996), [Online Los Alamos Archive Preprint]: cited on 8 Apr 1996, http://xxx.lanl. gov/abs/gr-qc/9604019. 2.2.2, 2.2.3

[130] Gundlach, C., "Critical Phenomena in Gravitational Collapse", Adv. Theor. Math. Phys., 2, 1-49, (1998). For a related online version see: C. Gundlach, "Critical Phenomena in Gravitational Collapse", (December, 1997), [Online Los Alamos Archive Preprint]: cited on 27 Jan 1998, http://xxx.lanl.gov/abs/gr-qc/9712084. [1, 2.2.1

Living Reviews in Relativity (2002-1)

http://www.livingreviews.org 
[131] Gundlach, C., "Nonspherical Perturbations of Critical Collapse and Cosmic Censorship", Phys. Rev. D, 57, 7075-7079, (1998). For a related online version see: C. Gundlach, "Nonspherical Perturbations of Critical Collapse and Cosmic Censorship", (October, 1997), [Online Los Alamos Archive Preprint]: cited on 12 Oct 1997, http://xxx.lanl.gov/abs/ gr-qc/9710066. 2.2.4

[132] Gundlach, C., and Martin-Garcia, J. M., "Charge Scaling and Universality in Critical Collapse", Phys. Rev. D, 54, 7353-7360, (1996). For a related online version see: C. Gundlach, et al., "Charge Scaling and Universality in Critical Collapse", (June, 1996), [Online Los Alamos Archive Preprint]: cited on 17 Sep 1996, http://xxx.lanl.gov/abs/gr-qc/9606072. 2.2.3

[133] Halpern, P., "Chaos in the Long-Term Behavior of Some Bianchi-type VIII Models", Gen. Relativ. Gravit., 19, 73-94, (1987). 3.1

[134] Hamadé, R. S., Horne, J. H., and Stewart, J. M., "Continuous SelfSimilarity and S-Duality", Class. Quantum Grav., 13, 2241-2254, (1996). For a related online version see: R. S. Hamadé, et al., "Continuous Self-Similarity and $S$-Duality", (November, 1995), [Online Los Alamos Archive Preprint]: cited on 7 Nov 1995, http://xxx.lanl.gov/abs/ gr-qc/9511024. 2.2.3

[135] Hamadé, R. S., and Stewart, J. M., "The Spherically Symmetric Collapse of a Massive Scalar Field", Class. Quantum Grav., 13, 497-512, (1996). For a related online version see: R. S. Hamadé, et al., "The Spherically Symmetric Collapse of a Massive Scalar Field", (June, 1995), [Online Los Alamos Archive Preprint]: cited on 22 Jun 1995, http://xxx.lanl.gov/ abs/gr-qc/9506044. [1, 2.2.1, 2.2.1

[136] Hara, T., Koike, T., and Adachi, S., "Renormalization Group and Critical Behavior in Gravitational Collapse", (July, 1996), [Online Los Alamos Archive Preprint]: cited on 28 May 1997, http://xxx.lanl.gov/abs/ gr-qc/9607010. 2.2.2, 2.2.4

[137] Harada, T., Iguchi, H., and Nakao, K., "Naked Singularity Explosion", Phys. Rev. D, 61, 101502, (2000). For a related online version see: T. Harada, et al., "Naked Singularity Explosion", (March, 2000), [Online Los Alamos Archive Preprint]: cited on 9 Mar 2000, http: //xxx.lanl.gov/abs/gr-qc/0003036. 2.1.4

[138] Harada, T., Iguchi, H., and Nakao, K., "Power, Energy, and Spectrum of a Naked Singularity Explosion", Phys. Rev. D, 62, 084037, (2000). For a related online version see: T. Harada, et al., "Power, Energy, and Spectrum of a Naked Singularity Explosion", (May, 2000), [Online Los Alamos Archive Preprint]: cited on 12 Jul 2000, http://xxx.lanl.gov/ abs/gr-qc/0005114. 2.1.4 
[139] Hawking, S. W., "The Occurrence of Singularities in Cosmology. III. Causality and Singularities", Proc. R. Soc. London, Ser. A, 300, 182201, (1967). 1

[140] Hawking, S. W., and Ellis, G. F. R., The Large Scale Structure of SpaceTime, (Cambridge University Press, Cambridge, 1973). [1]

[141] Hawking, S. W., and Penrose, R., "The Singularities of Gravitational Collapse and Cosmology", Proc. R. Soc. London, Ser. A, 314, 529-548, (1970). 1

[142] Hern, S. D., Numerical Relativity and Inhomogeneous Cosmologies, PhD Thesis, (Cambridge University, Cambridge, 2000). For a related online version see: S. D. Hern, "Numerical Relativity and Inhomogeneous Cosmologies", (April, 2000), [Online Los Alamos Archive Preprint]: cited on 12 Apr 2000, http://xxx.lanl.gov/gr-qc/0004036. 3.2.2

[143] Hern, S. J., and Stewart, J. M., "The Gowdy $T^{3}$ Cosmologies Revisited", Class. Quantum Grav., 15, 1581-1593, (1998). For a related online version see: S. J. Hern, et al., "The Gowdy $T^{3}$ Cosmologies Revisited", (August, 1997), [Online Los Alamos Archive Preprint]: cited on 5 May 1998, http: //xxx.lanl.gov/gr-qc/9708038. 3.2.2, 3.4.2

[144] Hirschmann, E. W., and Eardley, D. M., "Critical Exponents and Stability at the Black Hole Threshold for a Complex Scalar Field", Phys. Rev. D, 52, 5850-5856, (1995). For a related online version see: E. W. Hirschmann, et al., "Critical Exponents and Stability at the Black Hole Threshold for a Complex Scalar Field", (June, 1995), [Online Los Alamos Archive Preprint]: cited on 21 Aug 1995, http://xxx.lanl.gov/abs/gr-qc/ 9506078. ए2.2

[145] Hirschmann, E. W., and Eardley, D. M., "Universal Scaling and Echoing in Gravitational Collapse of a Complex Scalar Field", Phys. Rev. D, 51, 4198-4207, (1995). For a related online version see: E. W. Hirschmann, et al., "Universal Scaling and Echoing in Gravitational Collapse of a Complex Scalar Field", (December, 1994), [Online Los Alamos Archive Preprint]: cited on 21 Dec 1994, http://xxx.lanl.gov/abs/gr-qc/ 9412066. 2.2.1, 2.2.2

[146] Hirschmann, E. W., and Eardley, D. M., "Criticality and Bifurcation in the Gravitational Collapse of a Self-Coupled Scalar Field", Phys. Rev. D, 56, 4696-4705, (1997). For a related online version see: E. W. Hirschmann, et al., "Criticality and Bifurcation in the Gravitational Collapse of a SelfCoupled Scalar Field", (November, 1995), [Online Los Alamos Archive Preprint]: cited on 17 Nov 1995, http://xxx.lanl.gov/abs/gr-qc/ 9511052. 2.2.2, 2.2.3, 2.2.4

[147] Hobill, D. W., Bernstein, D., Welge, M., and Simkins, D., "The Mixmaster Cosmology as a Dynamical System", Class. Quantum Grav., 8, 1155-1171, (1991). [1, 3.3.1, 3.4.4

Living Reviews in Relativity (2002-1) http://www.livingreviews.org 
[148] Hobill, D. W., Burd, A., and Coley, A., eds., Deterministic Chaos in General Relativity, (Plenum, New York, 1994). 3.3.1

[149] Hobill, D. W., and Webster, P. S., "Trapped Surface Structure in Brill Wave Evolution", Talk presented at GR16 and private communication. [.].3

[150] Hod, S., "Radiative Tail of Realistic Rotating Gravitational Collapse", Phys. Rev. Lett., 84, 10-13, (2000). For a related online version see: S. Hod, "Radiative Tail of Realistic Rotating Gravitational Collapse", (July, 1999), [Online Los Alamos Archive Preprint]: cited on 2 Jan 2000, http://xxx.lanl.gov/abs/gr-qc/9907096. 2.3.3

[151] Hod, S., and Piran, T., "Critical Behavior and Universality in Gravitational Collapse of a Charged Scalar Field", Phys. Rev. D, 55, 3485-3496, (1997). For a related online version see: S. Hod, et al., "Critical Behaviour and Universality in Gravitational Collapse of a Charged Scalar Field", (July, 1996), [Online Los Alamos Archive Preprint]: cited on 31 Jul 1996, http://xxx.lanl.gov/abs/gr-qc/9606093. 2.2.3

[152] Hod, S., and Piran, T., "Fine-Structure of Choptuik's Mass-Scaling Relation", Phys. Rev. D, 55, 440-442, (1997). For a related online version see: S. Hod, et al., "Fine-Structure of Choptuik's Mass-Scaling Relation", (June, 1996), [Online Los Alamos Archive Preprint]: cited on 4 Jul 1996, http://xxx.lanl.gov/abs/gr-qc/9606087. 2.2.3

[153] Hod, S., and Piran, T., "The Inner Structure of Black Holes", Gen. Relativ. Gravit., 30, 1555-1562, (1998). For a related online version see: S. Hod, et al., "The Inner Structure of Black Holes", (February, 1999), [Online Los Alamos Archive Preprint]: cited on 2 Feb 1999, http://xxx.lanl.gov/abs/gr-qc/9902008. 2.3.2

[154] Hod, S., and Piran, T., "Mass Inflation in Dynamical Gravitational Collapse of a Charged Scalar Field", Phys. Rev. Lett., 81, 1554-1557, (1998). For a related online version see: S. Hod, et al., "Mass-Inflation in Dynamical Gravitational Collapse of a Charged Scalar-Field", (March, 1998), [Online Los Alamos Archive Preprint]: cited on 1 Mar 1998, http://xxx.lanl.gov/abs/gr-qc/9803004. 2.3.2

[155] Hübner, P., "How to Avoid Artificial Boundaries in the Numerical Calculation of Black Hole Spacetimes", (April, 1998), [Online Los Alamos Archive Preprint]: cited on 8 Mar 1999, http://xxx.lanl.gov/abs/ gr-qc/9804065. 2.3.3

[156] Hübner, P., "A Method for Calculating the Structure of (Singular) Spacetimes in the Large", Phys. Rev. D, 53, 701-721, (1996). For a related online version see: P. Hübner, "A Method for Calculating the Structure of (Singular) Spacetimes in the Large", (September, 1994), [Online Los 
Alamos Archive Preprint]: cited on 14 Sep 1994, http://xxx.lanl.gov/ abs/gr-qc/940902. 2.3.3

[157] Hübner, P., "Numerical Approach to the Global Structure of SpaceTimes", Helv. Phys. Acta, 69, 316-320, (1996). 2.3 .3

[158] Husa, S., Lechner, C., Pürrer, M., Thornburg, J., and Aichelburg, P. C., "Type II Critical Collapse of a Self-Gravitating Nonlinear $\sigma$ Model", Phys. Rev. D, 62, 104007, (2000). For a related online version see: S. Husa, et al., "Type II Critical Collapse of a Self-Gravitating Nonlinear $\sigma$ Model", (February, 2000), [Online Los Alamos Archive Preprint]: cited on 14 Sep 2000, http://xxx.lanl.gov/abs/gr-qc/0002067. 2.2.4

[159] Iguchi, H., and Harada, T., "Physical Aspects of Naked Singularity Explosion: How Does a Naked Singularity Explode?", Class. Quantum Grav., 18, 3681-3700, (2001). For a related online version see: H. Iguchi, et al., "Physical Aspects of Naked Singularity Explosion - How Does a Naked Singularity Explode? -", (July, 2001), [Online Los Alamos Archive Preprint]: cited on 31 Jul 2001, http://xxx.lanl.gov/abs/gr-qc/ 0107099. 2.I.

[160] Iguchi, H., Nakao, K., and Harada, T., "Gravitational Waves around a Naked Singularity: Odd-Parity Perturbation of Lemaître-Tolman-Bondi Space-Time", Phys. Rev. D, 57, 7262-7273, (1998). For a related online version see: H. Iguchi, et al., "Gravitational Waves around a Naked Singularity - Odd-Parity Perturbation of Lemaître-Tolman-Bondi Space-Time -", (April, 1998), [Online Los Alamos Archive Preprint]: cited on 6 Apr 1998, http://xxx.lanl.gov/abs/gr-qc/9804015. 2.1.4

[161] Iguchi, H., Nakao, K., and Harada, T., "Gravitational Waves around a Naked Singularity. II - Even-Parity Perturbation -", Prog. Theor. Phys., 103, 53-72, (2000). For a related online version see: H. Iguchi, et al., "Gravitational Waves around a Naked Singularity. II - Even-Parity Perturbation -", (April, 1999), [Online Los Alamos Archive Preprint]: cited on 4 Feb 2000, http://xxx.lanl.gov/abs/gr-qc/9911063. 2.1.4

[162] Iguchi, O., Hosoya, A., and Koike, T., "Renormalization Group Approach to the Einstein Equation in Cosmology", Phys. Rev. D, 57, 3340-3350, (1998). For a related online version see: O. Iguchi, et al., "Renormalization Group Approach to Einstein Equation in Cosmology", (September, 1997), [Online Los Alamos Archive Preprint]: cited on 16 Sep 1997, http: //xxx.lanl.gov/abs/gr-qc/9709042. 2.2.2

[163] Isenberg, J., and Kichenassamy, S., "Asymptotic Behavior in Polarized $T^{2}$-Symmetric Vacuum Spacetimes", J. Math. Phys., 40, 340-352, (1999). प, 3.4 .2

[164] Isenberg, J., and Moncrief, V., "Asymptotic Behavior in Polarized U(1)Symmetric Vacuum Spacetimes", Unpublished. 3.4.3

Living Reviews in Relativity (2002-1)

http://www.livingreviews.org 
[165] Isenberg, J. A., and Moncrief, V., "Asymptotic Behavior of the Gravitational Field and the Nature of Singularities in Gowdy Spacetimes", Ann. Phys. (N. Y.), 199, 84-122, (1990). [1, 3.1, 3.4.2

[166] Israel, W., "The Formation of Black Holes in Nonspherical Collapse and Cosmic Censorship", Can. J. Phys., 64, 120-127, (1986). 2.1.1

[167] Israel, W., "Must Nonspherical Collapse Produce Black Holes? A Gravitational Confinement Theorem", Phys. Rev. Lett., 56, 789-791, (1986). 2.1 .1

[168] Jantzen, R. T., "Finite-Dimensional Einstein-Maxwell-Scalar Field System", Phys. Rev. D, 33, 2121-2135, (1986). 3.1, 3.3.1, 3.4.1

[169] Jantzen, R. T., "Spatially Homogeneous Dynamics: A Unified Picture", in Ruffini, R., and Melchiorri, F., eds., Gamov Cosmology, 61-147, (North Holland, Amsterdam, 1987). For a related online version see: R. T. Jantzen, "Spatially Homogeneous Dynamics: A Unified Picture", (February, 2001), [Online Los Alamos Archive Preprint]: cited on 12 Feb 2001, http://xxx.lanl.gov/abs/gr-qc/0102035. 3.3.1, 3.4.2

[170] Johnson, G., "What a Physicist Finds Obscene", The New York Times, (16 Feb 1997). 2.2.1

[171] Kasner, E., "Solutions of the Einstein Equations Involving Functions of Only One Variable", Trans. Am. Math. Soc., 27, 155-162, (1925). 3.1, 5.3.

[172] Khalatnikov, I. M., Lifshitz, E. M., Khanin, K. M., Shchur, L. N., and Sinai, Ya. G., "On the Stochasticity in Relativistic Cosmology", J. Stat. Phys., 38, 97-114, (1985). 3.3.1

[173] Kichenassamy, S., and Rendall, A. D., "Analytic Description of Singularities in Gowdy Spacetimes", Class. Quantum Grav., 15, 1339-1355, (1998). 廿, 3.4 .2

[174] Kirillov, A. A., "The Nature of the Spatial Distribution of Metric Inhomogeneities in the General Solution of the Einstein Equations near a Cosmological Singularity", JETP, 76, 355-358, (1993). 3.4.1

[175] Kirillov, A. A., and Kochnev, A. A., "Cellular Structure of Space near a Singularity in Time in Einstein's Equations", JETP Lett., 46, 435-438, (1987). 3.4 .1

[176] Koike, T., Hara, T., and Adachi, S., "Critical Behavior in Gravitational Collapse of Radiation Fluid: A Renormalization Group (Linear Perturbation) Analysis", Phys. Rev. Lett., 74, 5170-5173, (1995). 2.2 .2

[177] LeBlanc, V. G., "Asymptotic States of Magnetic Bianchi I Cosmologies", Class. Quantum Grav., 14, 2281-2301, (1997). 3.1, 3.3.1, 3.4.1

Living Reviews in Relativity (2002-1) http://www.livingreviews.org 
[178] LeBlanc, V. G., Kerr, D., and Wainwright, J., "Asymptotic States of Magnetic Bianchi $\mathrm{VI}_{0}$ Cosmologies", Class. Quantum Grav., 12, 513-541, (1995). 3.1, 3.3.1, 3.4.1

[179] Libson, J., Masso, J., Seidel, E., Suen, W.-M., and Walker, P., "Event Horizons in Numerical Relativity I: Methods and Tests", Phys. Rev. D, 53, 4335-4350, (1996). For a related online version see: J. Libson, et al., "Event Horizons in Numerical Relativity I: Methods and Tests", (December, 1994), [Online Los Alamos Archive Preprint]: cited on 22 Dec 1994, http://xxx.lanl.gov/abs/gr-qc/9412068. 2.1.1, 2.1.4

[180] Liebling, S. L., Hirschmann, E. W., and Isenberg, J., "Critical Phenomena in Nonlinear Sigma Models", J. Math. Phys., 41, 5691-5700, (2000). For a related online version see: S. L. Liebling, et al., "Critical Phenomena in Nonlinear Sigma Models", (November, 1999), [Online Los Alamos Archive Preprint]: cited on 1 Jun 2000, http://xxx.lanl.gov/abs/math-ph/ 9911020. 2.2.4

[181] Ma, P. K.-H., and Wainwright, J., "A Dynamical Systems Approach to the Oscillatory Singularity in Bianchi Cosmologies", in Hobill, D. W., Burd, A., and Coley, A., eds., Deterministic Chaos in General Relativity, (Plenum, New York, 1994). 3.3 .1

[182] MacCallum, M., "Anisotropic and Inhomogeneous Relativistic Cosmologies", in Hawking, S. W., and Israel, W., eds., General Relativity, an Einstein Centenary Survey, chapter 11, (Cambridge University, Cambridge, 1979). 3.4.1

[183] Maison, D., "Non-Universality of Critical Behavior in Spherically Symmetric Gravitational Collapse", Phys. Lett. B, 366, 82-84, (1966). For a related online version see: D. Maison, "Non-Universality of Critical Behavior in Spherically Symmetric Gravitational Collapse", (April, 1995), [Online Los Alamos Archive Preprint]: cited on 05 Apr 1995, http://xxx.lanl.gov/abs/gr-qc/9504008. 2.2.2

[184] Masso, J., Seidel, E., Suen, W.-M., and Walker, P., "Event Horizons in Numerical Relativity II: Analyzing the Horizon", Phys. Rev. D, 59, 064015, (1999). For a related online version see: J. Masso, et al., "Event Horizons in Numerical Relativity II: Analyzing the Horizon", (April, 1998), [Online Los Alamos Archive Preprint]: cited on 23 Apr 1998, http://xxx.lanl.gov/abs/gr-qc/9804059. 2.1.1, 2.1.4

[185] Mellor, F., and Moss, I., "Stability of Black Holes in de Sitter Space", Phys. Rev. D, 41, 403-409, (1990). 2.3.3

[186] Mellor, F., and Moss, I., "A Reassessment of the Stability of the Cauchy Horizon in de Sitter Space", Class. Quantum Grav., 9, L43-L46, (1992). 2.3 .3

Living Reviews in Relativity (2002-1)

http://www.livingreviews.org 
[187] Misner, C. W., "Mixmaster Universe", Phys. Rev. Lett., 22, 1071-1074, (1969). [1, 3.1, 3.3.1

[188] Moncrief, V., "Global Properties of Gowdy Spacetimes with $T^{3} \times R$ Topology", Ann. Phys. (N. Y.), 132, 87-107, (1981). 3.4 .2

[189] Moncrief, V., "Finite-Difference Approach to Solving Operator Equations of Motion in Quantum Theory", Phys. Rev. D, 28, 2485-2490, (1983). 5.2.

[190] Moncrief, V., "Reduction of Einstein's Equations for Vacuum Space-Times with Spacelike $U(1)$ Isometry Groups", Ann. Phys. (N. Y.), 167, 118-142, (1986). 3.4.1, 3.4.3

[191] Moncrief, V., "Spacetime Singularities and Cosmic Censorship", in Francaviglia, M., Longhi, G., Lusanna, L., and Sorace, E., eds., General Relativity and Gravitation, 259-276, (World Scientific, Singapore, 1997). [1

[192] Montani, G., "On the General Behavior of the Universe near the Cosmological Singularity", Class. Quantum Grav., 12, 2505-2517, (1995). 3.4.1

[193] Moser, A. A., Matzner, R. A., and Ryan, Jr., M. P., "Numerical Solutions for Symmetric Bianchi Type IX Universes", Ann. Phys. (N. Y.), 79, 558579, (1973). 1, 3.3.1

[194] Motter, A. E., and Letelier, P. S., "Mixmaster Chaos", Phys. Lett. A, 285, 127-131, (2001). For a related online version see: A. E. Motter, et al., "Mixmaster Chaos", (November, 2000), [Online Los Alamos Archive Preprint]: cited on 1 Nov 2000, http://xxx.lanl.gov/abs/gr-qc/ 0011001. 田, 3.1, 3.3.1

[195] Nakamura, T., and Sato, H., "General Relativistic Collapse of NonRotating Axisymmetric Stars", Prog. Theor. Phys., 67, 1396-1405, (1982). 2.1 .2

[196] Nakamura, T., Shapiro, S. L., and Teukolsky, S. A., "Naked Singularities and the Hoop Conjecture: An Analytic Exploration", Phys. Rev. D, 38, 2972-2978, (1988). 2.1 .2

[197] Nakamura, T., Shibata, M., and Nakao, K., "Naked Singularity Dries Up?", Prog. Theor. Phys., 89, 821-831, (1993). 2.1.4

[198] Nakao, K., Iguchi, H., and Harada, T., "Newtonian Analysis of Gravitational Waves from Naked Singularity", Phys. Rev. D, 63, 084003, (2001). For a related online version see: K. Nakao, et al., "Newtonian Analysis of Gravitational Waves from Naked Singularity", (June, 2000), [Online Los Alamos Archive Preprint]: cited on 16 Jun 2000, http://xxx.lanl.gov/abs/astro-ph/0006057. 2.1.4 
[199] Niemeyer, J. C., and Jedamzik, K., "Near-Critical Gravitational Collapse and the Initial Mass Function of Primordial Black Holes", Phys. Rev. Lett., 80, 5481-5484, (1998). For a related online version see: J. C. Niemeyer, et al., "Near-Critical Gravitational Collapse and the Initial Mass Function of Primordial Black Holes", (September, 1997), [Online Los Alamos Archive Preprint]: cited on 9 Sep 1997, http://xxx.lanl.gov/abs/astro-ph/ 9709072. 2.2.3

[200] Norton, A. H., "Finite Difference Operators for PDE's Based on Sampling Kernels for Spline Quasi-Interpolation", University of New South Wales Preprint, (1992). 3.4 .3

[201] Olabarrieta, I., Critical Collapse of Collisionless Matter in Spherical Symmetry, Masters Thesis, (Uinversity of British Columbia, Vancouver, 2000). For a related online version see: I. Olabarrieta, "Critical Collapse of Collisionless Matter in Spherical Symmetry", (December, 2000), [Online Los Alamos Archive Preprint]: cited on 17 Dec 2000, http://xxx.lanl.gov/abs/gr-qc/0012059. 2.2.3

[202] Ori, A., "Inner Structure of a Charged Black Hole: An Exact MassInflation Solution", Phys. Rev. Lett., 67, 789-792, (1991). 1, 2.3.1, 2.3 .2 , 2.3.2

[203] Ori, A., "Structure of the Singularity inside a Realistic Black Hole", Phys. Rev. Lett., 68, 2117-2120, (1992). 2.3.1, 2.3.2, 2.3.2

[204] Ori, A., "Null Weak Singularities in Plane-Symmetric Spacetimes", Phys. Rev. D, 57, 4745-4753, (1998). For a related online version see: A. Ori, "Null Weak Singularities in Plane-Symmetric Spacetimes", (January, 1998), [Online Los Alamos Archive Preprint]: cited on 26 Jan 1998, http://xxx.lanl.gov/abs/gr-qc/9801086. 2.3.2

[205] Ori, A., "Evolution of Linear Gravitational and Electromagnetic Perturbations inside a Kerr Black Hole", Phys. Rev. D, 61, 024001, (1999). 2.3.

[206] Ori, A., "Oscillatory Null Singularity inside Realistic Spinning Black Holes", Phys. Rev. Lett., 83, 5423-5426, (1999). For a related online version see: A. Ori, "Oscillatory Null Singularity inside Realistic Spinning Black Holes", (January, 2001), [Online Los Alamos Archive Preprint]: cited on 5 Mar 2001, http://xxx.lanl.gov/abs/gr-qc/0103012. 2.3.2

[207] Ove, R., "Nonlinear Gravitational Effect", Phys. Rev. Lett., 64, 12001203, (1990). 3.4.4

[208] Pelath, M. A., Tod, K. P., and Wald, R. M., "Trapped Surfaces in Prolate Collapse in the Gibbons-Penrose Construction", Class. Quantum Grav., 15, 3917-3934, (1998). For a related online version see: M. A. Pelath, et

Living Reviews in Relativity (2002-1)

http://www.livingreviews.org 
al., "Trapped Surfaces in Prolate Collapse in the Gibbons-Penrose Construction", (May, 1998), [Online Los Alamos Archive Preprint]: cited on 13 May 1998, http://xxx.lanl.gov/abs/gr-qc/9805051. 2.1.3, 2]

[209] Penrose, R., "Gravitational Collapse: The Role of General Relativity", Riv. Nuovo Cimento, 1, 252-276, (1969). 2

[210] Penrose, R., "Singularities and Time Asymmetry", in Hawking, S. W., and Israel, W., eds., General Relativity: An Einstein Centenary Survey, (Cambridge University Press, Cambridge, 1979). 2

[211] Poisson, E., "Black-Hole Interiors and Strong Cosmic Censorship", in Burko, L. M., and Ori, A., eds., Internal Structure of Black Holes and Spacetime Singularities, (Institute of Physics, Bristol, 1998). For a related online version see: E. Poisson, "Black-Hole Interiors and Strong Cosmic Censorship", (September, 1997), [Online Los Alamos Archive Preprint]: cited on 10 Sep 1997, http://xxx.lanl.gov/abs/gr-qc/9709022. 2.3.3

[212] Poisson, E., and Israel, W., "Inner-Horizon Instability and Mass Inflation in Black Holes", Phys. Rev. Lett., 63, 1663-1666, (1989). 1, 2.3.1, 2.3.2

[213] Poisson, E., and Israel, W., "Internal Structure of Black Holes", Phys. Rev. D, 41, 1796-1807, (1990). 2.3.1, 2.3.2

[214] Press, W. H., Flannery, B. P., Teukolsky, S. A., and Vetterling, W. T., Numerical Recipes: the Art of Scientific Computing (2nd edition), (Cambridge University, Cambridge, 1992). 3.3.1

[215] Pretorius, F., and Choptuik, M. W., "Gravitational Collapse in $2+1$ Dimensional AdS Spacetime", Phys. Rev. D, 62, 124012, (2000). For a related online version see: F. Pretorius, et al., "Gravitational Collapse in 2+1 Dimensional AdS Spacetime", (July, 2000), [Online Los Alamos Archive Preprint]: cited on 6 Nov 2000, http://xxx.lanl.gov/abs/ gr-qc/0007008. 2.2.4

[216] Pullin, J., "Time and Chaos in General Relativity", in SILARG VII Relativity and Gravitation: Classical and Quantum, (World Scientific, Singapore, 1991). 1, 3.3.1

[217] Rein, G., Rendall, A. D., and Schaeffer, J., "Critical Collapse of Collisionless Matter: a Numerical Investigation", Phys. Rev. D, 58, 044007, (1998). For a related online version see: G. Rein, et al., "Critical Collapse of Collisionless Matter - a Numerical Investigation", (April, 1998), [Online Los Alamos Archive Preprint]: cited on 17 Apr 1998, http: //xxx.lanl.gov/abs/gr-qc/9804040. 2.2.3

[218] Rendall, A. D., "Global Dynamics of the Mixmaster Model", Class. Quantum Grav., 14, 2341-2356, (1997). For a related online version see: A. D. Rendall, "Global Dynamics of the Mixmaster Model", (March, 
1997), [Online Los Alamos Archive Preprint]: cited on 14 Mar 1997, http://xxx.lanl.gov/abs/gr-qc/9703036. 3.3.1, 3.3.2

[219] Rendall, A. D., "Solutions of the Einstein Equations with Matter", in Francaviglia, M., Longhi, G., Lusanna, L., and Sorace, E., eds., General Relativity and Gravitation, 313-336, (World Scientific, Singapore, 1997). For a related online version see: A. D. Rendall, "Solutions of the Einstein Equations with Matter", (October, 1995), [Online Los Alamos Archive Preprint]: cited on 5 Oct 1995, http://xxx.lanl.gov/abs/ gr-qc/9510009. [

[220] Rendall, A. D., and Weaver, M., "Manufacture of Gowdy Spacetimes with Spikes", Class. Quantum Grav., 18, 2959-2976, (2001). For a related online version see: A. D. Rendall, et al., "Manufacture of Gowdy Spacetimes with Spikes", (March, 2001), [Online Los Alamos Archive Preprint]: cited on 28 Mar 2001, http://xxx.lanl.gov/abs/gr-qc/0103102. 3.4.2

[221] Ringström, H., "Curvature Blow up in Bianchi VIII and IX Vacuum Spacetimes", Class. Quantum Grav., 17, 713-731, (2000). For a related online version see: H. Ringström, "Curvature Blow up in Bianchi VIII and IX Vacuum Spacetimes", (November, 1999), [Online Los Alamos Archive Preprint]: cited on 29 Nov 1999, http://xxx.lanl.gov/abs/gr-qc/ 9911115. उ.3.2

[222] Ringström, H., "The Bianchi IX Attractor", Ann. Henri Poincare, 2, 405500, (2001). For a related online version see: H. Ringström, "The Bianchi IX Attractor", (June, 2000), [Online Los Alamos Archive Preprint]: cited on 10 Jun 2000, http://xxx.lanl.gov/abs/gr-qc/0006035. 3.3 .2

[223] Rugh, S. E., Chaotic Behavior and Oscillating Three-Volumes in a SpaceTime Metric in General Relativity, Masters Thesis, (Niels Bohr Institute, Copenhagen, 1990). Available on request to the author. [1, 3.3.1

[224] Rugh, S. E., "Chaos in the Einstein Equations - Characterization and Importance?", in Hobill, D. W., Burd, A., and Coley, A., eds., Deterministic Chaos in General Relativity, (Plenum, New York, 1994). 3.3.1

[225] Rugh, S. E., and Jones, B. J. T., "Chaotic Behaviour and Oscillating Three-Volumes in Bianchi IX Universes", Phys. Lett. A, 147, 353, (1990). प, 3.3.1

[226] Ryan Jr., M. P., "Qualitative Cosmology: Diagrammatic Solutions for Bianchi IX Universes with Expansion, Rotation, and Shear II: The General Case", Ann. Phys. (N. Y.), 68, 541-555, (1971). 3.3 .1

[227] Ryan Jr., M. P., and Shepley, L. C., Homogeneous Relativistic Cosmologies, (Princeton University, Princeton, 1975). 3.1, 3.4.2

[228] Schoen, R., and Yau, S.-T., "The Existence of a Black Hole Due to Condensation of Matter", Commun. Math. Phys., 90, 575-579, (1983). 2.1.1

Living Reviews in Relativity (2002-1)

http://www.livingreviews.org 
[229] Seidel, E., and Suen, W.-M., "Formation of Solitonic Stars Through Gravitational Cooling", Phys. Rev. Lett., 72, 2516-2519, (1994). For a related online version see: E. Seidel, et al., "Formation of Solitonic Stars Through Gravitational Cooling", (September, 1993), [Online Los Alamos Archive Preprint]: cited on 14 Sep 1993, http://xxx.lanl.gov/abs/ gr-qc/9309015. 2.2.3

[230] Shapiro, S. L., and Teukolsky, S. A., "Formation of Naked Singularities: The Violation of Cosmic Censorship", Phys. Rev. Lett., 66, 994-997, (1991). 2.1.2, 2.1.4

[231] Shapiro, S. L., and Teukolsky, S. A., "Gravitational Collapse of Rotating Spheroids and the Formation of Naked Singularities", Phys. Rev. D, 45, 2006-2012, (1992). 2.1.2

[232] Shapiro, S. L., and Teukolsky, S. A., "Relativistic Stellar Systems with Spindle Singularities", Astrophys. J., 419, 622-635, (1993). 2.1 .2

[233] Stahl, F., "Fuchsian Analysis of $S^{2} \times S^{1}$ and $S^{3}$ Gowdy Spacetimes", (September, 2001), [Online Los Alamos Archive Preprint]: cited on 4 Sep 2001, http://xxx.lanl.gov/abs/gr-qc/0109011. 3.4.2

[234] Suzuki, M., "Fractal Decomposition of Exponential Operators with Applications to Many-body Theories and Monte Carlo Simulations", Phys. Lett. A, 146, 319-323, (1990). 3.2.1

[235] Suzuki, M., "General Theory of Fractal Path Integrals with Applications to Many-body Theories and Statistical Physics", J. Math. Phys., 32, 400407, (1991). 3.2.1

[236] Taub, A., "Empty Space-Times Admitting a Three-Parameter Group of Motions", Ann. Math., 53, 472, (1951). 3.1, 3.3.1

[237] Teukolsky, S. A., "Stability of the Iterated Crank-Nicholson Method in Numerical Relativity", Phys. Rev. D, 61, 087501, (2000). For a related online version see: S. A. Teukolsky, "On the Stability of the Iterated Crank-Nicholson Method in Numerical Relativity", (September, 1999), [Online Los Alamos Archive Preprint]: cited on 7 Sep 1999, http:// xxx.lanl.gov/abs/gr-qc/9909026. 3.2.2

[238] Thornburg, J., Lechner, C., Purrer, M., Aichelburg, P. C., and Husa, S., "Episodic Self-Similarity in Critical Gravitational Collapse", (December, 2000), [Online Los Alamos Archive Preprint]: cited on 22 May 2001, http://xxx.lanl.gov/abs/gr-qc/0012043. 2.2.4

[239] Thorne, K. S., "Nonspherical Gravitational Collapse - A Short Review", in Klauder, J., ed., Magic without Magic, 231-258, (W. H. Freeman, San Francisco, 1972). 1, 2.1.1, 2.1.4

Living Reviews in Relativity (2002-1) http://www.livingreviews.org 
[240] Tipler, F. J., Clarke, C. J. S., and Ellis, G. F. R., "Singularities and Horizons - A Review Article", in Held, A., ed., General Relativity and Gravitation, 97-206, (Plenum, New York, 1980). [1

[241] Tod, K. P., "The Hoop Conjecture and the Gibbons-Penrose Construction of Trapped Surfaces", Class. Quantum Grav., 9, 1581-1591, (1992). 2.1.2, 2.T.3

[242] van Elst, H., Uggla, C., and Wainwright, J., "Dynamical Systems Approach to $G_{2}$ Cosmology", (July, 2001), [Online Los Alamos Archive Preprint]: cited on 9 Nov 2001, http://xxx.lanl.gov/abs/gr-qc/ 0107041. ए

[243] van Putten, M. H. P. M., "Numerical Integration of Nonlinear Wave Equations for General Relativity", Phys. Rev. D, 55, 4705-4711, (1997). For a related online version see: M. H. P. M. van Putten, "Numerical Integration of Nonlinear Wave Equations for General Relativity", (January, 1997), [Online Los Alamos Archive Preprint]: cited on 10 Jan 1997, http://xxx.lanl.gov/abs/gr-qc/9701019. 3.4.2

[244] Wald, R. M., "Gravitational Collapse and Cosmic Censorship", (October, 1997), [Online Los Alamos Archive Preprint]: cited on 6 Nov 1997, http: //xxx.lanl.gov/abs/gr-qc/9710068. [1, 2, 2.1.1, 2.1.2

[245] Wald, R. M., General Relativity, (University of Chicago Press, Chicago, 1984). 1, 2.3.1

[246] Wald, R. M., and Iyer, V., "Trapped Surfaces in the Schwarzschild Geometry and Cosmic Censorship", Phys. Rev. D, 44, 3719-3722, (1991). 2.1 .1 , 2.1.2, 2.1.3

[247] Weaver, M., Asymptotic Behavior to Solutions to Einstein's Equation, $\mathrm{PhD}$ Thesis, (University of Oregon, Eugene, 1999). 3.4.1, 3.4.2

[248] Weaver, M., "Dynamics of Magnetic Bianchi VI ${ }_{0}$ Cosmologies", Class. Quantum Grav., 17, 421-434, (2000). For a related online version see: M. Weaver, "Dynamics of Magnetic Bianchi $\mathrm{VI}_{0}$ Cosmologies", (September, 1999), [Online Los Alamos Archive Preprint]: cited on 11 Nov 1999, http://xxx.lanl.gov/abs/gr-qc/9909043. 3.3.2, 3.4.1

[249] Weaver, M., Berger, B. K., and Isenberg, J., "Oscillatory Approach to the Singularity in Vacuum $T^{2}$ Symmetric Spacetimes", in Gurzadyan, V., and Jantzen, R. T., eds., 9th Marcel Grossmann Meeting, (World Scientific, Singapore, 2002). For a related online version see: M. Weaver, et al., "Oscillatory Approach to the Singularity in Vacuum $T^{2}$ Symmetric Spacetimes", (January, 2001), [Online Los Alamos Archive Preprint]: cited on 16 Jan 2001, http://xxx.lanl.gov/abs/gr-qc/0101054. 3.4.2

Living Reviews in Relativity (2002-1)

http://www.livingreviews.org 
[250] Weaver, M., Isenberg, J., and Berger, B. K., "Mixmaster Behavior in Inhomogeneous Cosmological Spacetimes", Phys. Rev. Lett., 80, 29842987, (1998). For a related online version see: M. Weaver, et al., "Mixmaster Behavior in Inhomogeneous Cosmological Spacetimes", (December, 1997), [Online Los Alamos Archive Preprint]: cited on 11 Mar 1998, http://xxx.lanl.gov/abs/gr-qc/9712055. [1, 3.4.1, 3.4.2

[251] Wojtkiewicz, J., "Naked Singularities in Initial Surfaces", Phys. Rev. D, 41, 1867-1874, (1990). 2.1 .2

[252] Yokoyama, J., "Cosmological Constraints on Primordial Black Holes Produced in Near-Critical Gravitational Collapse", Phys. Rev. D, 58, 107502, (1998). For a related online version see: J. Yokoyama, "Cosmological Constraints on Primordial Black Holes Produced in Near-Critical Gravitational Collapse", (April, 1998), [Online Los Alamos Archive Preprint]: cited on 24 Jul 1998, http://xxx.lanl.gov/abs/gr-qc/9804041. 2.2.3

[253] Zardecki, A., "Modeling in Chaotic Relativity", Phys. Rev. D, 28, 12351242, (1983). 3.4.4

[254] Zotov, M., "Einstein-Yang-Mills Black Hole Interiors: Serious Problems But Simple Solution", (April, 1997), [Online Los Alamos Archive Preprint]: cited on 11 Jun 1997, http://xxx.lanl.gov/ps/gr-qc/ 9704080. ए.3.2 